TOPICAL REVIEW

\title{
Quantum computing with quantum-Hall edge state interferometry
}

To cite this article: Paolo Bordone et al 2019 Semicond. Sci. Technol. 34103001

View the article online for updates and enhancements.

\section{IOP ebooks}

Bringing you innovative digital publishing with leading voices

to create your essential collection of books in STEM research.

Start exploring the collection - download the first chapter of every title for free. 


\title{
Topical Review
}

\section{Quantum computing with quantum-Hall edge state interferometry}

\author{
Paolo Bordone ${ }^{1,2}{ }^{(1)}$, Laura Bellentani ${ }^{1}$ (i) and Andrea Bertoni ${ }^{2}$ (i) \\ ${ }^{1}$ Dipartimento di Scienze Fisiche, Informatiche e Matematiche, Università degli Studi di Modena e Reggio \\ Emilia, Via Campi 213/A, I-41125, Modena, Italy \\ ${ }^{2}$ S3, Istituto Nanoscienze-CNR, Via Campi 213/A, I-41125, Modena, Italy \\ E-mail: paolo.bordone@unimore.it
}

Received 27 July 2018, revised 6 August 2019

Accepted for publication 16 August 2019

Published 16 September 2019

\begin{abstract}
Electron interferometers based on Hall edge states (ESs) proved to be robust demonstrators of the coherent quantum dynamics of carriers. Several proposals to expose their capability to build and control quantum entanglement and to exploit them as building block for quantum computing devices has been presented. Here, we review the time-dependent numerical modeling of Hall interferometers operating at the single-carrier level at integer filling factor (FF). By defining the qubit state either as the spatial localization (at FF 1) or the Landau index (at FF 2) of a single carrier propagating in the ES, we show how a generic one-qubit rotation can be realized. By a proper design of the two-dimensional electron gas potential landscape, an entangling two-qubit gate can be implemented by exploiting Coulomb interaction, thus realizing a universal set of quantum gates. We also assess how the shape of the edge confining potential affects the visibility of the quantum transformations.
\end{abstract}

Keywords: quantum computing, edge states, electron interferometry

(Some figures may appear in colour only in the online journal)

\section{Introduction}

Devices based on coherent carrier transport in semiconductor nanostructures play a strategic role in the quest for viable systems able to process quantum information, due to their intrinsic scalability and integrability with conventional electronics. This approach is usually termed flying-qubit quantum computation $[1,2]$, as the quantum bit of information is encoded in the carrier state, which propagates along the device to interact with other qubits.

However, carrier states in nanostructured semiconductors, as quantum wires or quantum dots, proved to be highly vulnerable to decoherence. A possible solution consists in encoding the qubit in a topologically-protected state, immune to decoherence, at least in an ideal system. Edge states (ESs) of a semiconductor subject to a magnetic field, thus operating in the quantum Hall regime, are a stereotypical example of topologically protected states, and function as ideal quasi-1D transport channels where electrons propagate protected from several types of scattering processes [3].

If ESs are able to guarantee the degree of quantum coherence needed for the serial operation of several quantum gates, is still an open question. The first attempts to measure the coherence length of electrons in edge channels were based on quantum dots in the integer quantum Hall (IQH) regime [4], coupled to a source and a drain lead by consecutive narrow constrictions forming two quantum point contacts (QPCs). Two ESs form two closed loop channels inside the dot, one of which is separated in a transmitted and a reflected path by each QPCs. As a consequence, in this kind of device, electrons can tunnel through two QPCs from the source to the drain, while inside the quantum dot the path of the outer ES can be closed in a loop and isolated to define confined states. Magnetically-driven Aharonov-Bohm (AB) oscillations in the resistance thus arise as an effect of the electron selfinterference. However, these are affected by the interplay with 
Coulomb-blockade effects [5] or energy averaging due to thermal smearing [4]. The first clear measurement of the electron coherence length in edge channels was reported in [6], based on an electron Mach-Zehnder interferometer (MZI) operating at bulk filling factor (FF) 2. Differently from the quantum dot setup, in the latter device the path of the two interfering edge channels could be tuned by top gates, and the path mismatch could be reduced to zero to avoid thermal smearing. By assessing the visibility of the electronic MZI, which is related to the coherence length, a result of $20 \mu \mathrm{m}$ was obtained for a device temperature of $20 \mathrm{mK}$.

Earliest experimental implementations of these devices rely upon the injection of stationary currents $[7,8]$, so that the numerical characterization of the electron dynamics in ESs is generally afforded through scattering states. However, the realization of quantum logic gates able to process the information encoded in a single particle has been made possible thanks to the recent availability of single-electron sources and new excitation techniques, that allow for injecting localized wavepackets inside ESs [9, 10]. Numerical models able to account for electron localization in a dynamical framework are therefore fundamental for a proper modeling of such devices.

The coherence of ES channels enables quantum optics experiments where electrons play the analogous role of single photons emitted in an optical medium [11]. The major differences are the Pauli exclusion principle, that allows a highly controlled autocorrelation and noise-free stream of electrons, and the strong coulomb interaction that favours phase manipulation and the control of entanglement strength in fewelectron implementations. The controlled emission of singleelectron excitations constitutes also an important tool for the study of exotic statistics, as for anyons in the fractional quantum Hall effect [12], to probe interactions in 1D systems by time of flight measurements, as for charge fractionalization in the IQH regime [13], and possibly to realize ultra-fast quantum nanoelectronics.

Here, we review recent proposals for the realization of quantum gates with ESs in the Hall regime at integer FF and results showing the capability of controlling the occupation of ESs up to the level of single carriers [14]. Time-dependent simulations of single-carrier interferometers based on III-V materials indicate the viability of this flying qubit approach, constituting a substantial improvement with respect to earlier proposals based on ballistic electrons in coupled quantum wires [1, 2, 15-21]. Specifically, two different proposals will be illustrated, operating at FF 1 and 2, with the qubit defined as either the ES occupancy or the Landau level (LL) of the electron, respectively. We will show that the geometry at FF 2 favors the miniaturization of the device and a higher visibility. It also solves the intrinsic non-scalability problem of the first Hall interferometers, thus allowing their concatenation in many-qubit architectures. By resorting to this geometry, we will indeed describe and simulate devices with two interacting electrons, that are able to manipulate two-electron states for quantum information protocols [22, 23]. These can be exploited, in principle, to measure the indistinguishability of particles generated from different sources [24] or to devise quantum computation logic gates based on entangled qubits, as for superconducting Cooper pairs [25] or in micromaser set-ups [26].

This work is organized as follows. Section 2 describes the general notation for the quantum transformations used in the following. Section 3 drafts the basic theory of quantum Hall ESs and introduces the form of the localized wave packets (WPs) used as initial condition for the time-dependent numerical simulations. In section 4 , the modeling of quantum interferometers based on Hall ESs is presented, with a specific focus on the functioning of the beam splitters (BSs). In section 5, the numerical method is described, and the numerical results of the single-carrier dynamics are rationalized. Section 6 describes the two-particle dynamics with an exact simulation of the Pauli dip in an electronic Hong-Ou-Mandel interferometer. Finally, conclusions are drawn in section 7.

\section{Quantum gates with flying qubits}

This section is devoted to a short description of basic principles and notation of quantum computing and to the introduction of the matrix representation of quantum logic gates. In particular, we are interested in the so-called charge flying qubits model [27-29], where the elementary bits of quantum information (the qubit) move synchronously along predetermined paths and undergo single-qubit operations or, through an effective mutual interaction, two-qubit transformations. Although the notation is general in the framework of quantum information processing, we will refer to our system of interest, with the qubit represented by a single carrier moving along one or a couple of ESs. Furthermore, we will limit our description to quantum gates that will be numerically modeled in the next sections.

The interest in quantum information theory is currently an exceptionally fertile research topics, with several branches like quantum algorithms, quantum cryptography, quantum communication, quantum teleportation [30] and, finally, quantum computation. Indeed, the latter topic represents the holy grail of quantum information processing, since the realization of a robust, affordable and scalable architecture able to implement universal quantum computation would have an impact on society that is difficult to overestimate.

At the present time, two different technology platforms have demonstrated the clear capability to achieve multi-qubit operation in a controlled and possibly scalable way. They are based on a fully connected trapped-ion network [31] and on superconducting charge qubits [32]. However, the quest for alternative approaches is still very active, especially in the field of semiconductor nanoelectronics, including devices based on commercial CMOS technology [33]. The system analyzed in this work, namely flying qubits encoded by the states of carriers propagating in ESs, seems a promising candidate due to its robustness against decoherence and its integrability with traditional semiconductor technology.

The two possible states of a qubit, namely $|0\rangle$ and $|1\rangle$, are the standard basis on which the single-qubit gates are represented. A general transformation of one qubit is indicated by a 
rotation matrix of the $S U(2)$ group, i.e.

$\mathbf{U}(\alpha, \beta, \theta)=\left(\begin{array}{cc}\mathrm{e}^{\mathrm{i}(\alpha / 2+\beta / 2)} \cos \left(\frac{\theta}{2}\right) & \mathrm{e}^{\mathrm{i}(\alpha / 2-\beta / 2)} \sin \left(\frac{\theta}{2}\right) \\ -\mathrm{e}^{\mathrm{i}(-\alpha / 2+\beta / 2)} \sin \left(\frac{\theta}{2}\right) & \mathrm{e}^{\mathrm{i}(-\alpha / 2-\beta / 2)} \cos \left(\frac{\theta}{2}\right)\end{array}\right)$,

where $\alpha, \beta, \theta$ are three real numbers representing three rotation angles around two orthogonal axes of the corresponding pseudo-spin state. Any transformation $\mathbf{U}$ can be obtained using two of the three basic rotations:

$$
\mathbf{R}_{\mathbf{x}}(\theta)=\left(\begin{array}{cc}
\cos \left(\frac{\theta}{2}\right) & \mathrm{i} \sin \left(\frac{\theta}{2}\right) \\
\mathrm{i} \sin \left(\frac{\theta}{2}\right) & \cos \left(\frac{\theta}{2}\right)
\end{array}\right),
$$

around the $X$ axis,

$$
\mathbf{R}_{\mathbf{y}}(\theta)=\left(\begin{array}{cc}
\cos \left(\frac{\theta}{2}\right) & \sin \left(\frac{\theta}{2}\right) \\
-\sin \left(\frac{\theta}{2}\right) & \cos \left(\frac{\theta}{2}\right)
\end{array}\right)
$$

around the $Y$ axis, and

$$
\mathbf{R}_{\mathbf{z}}(\theta)=\left(\begin{array}{cc}
\mathrm{e}^{\mathrm{i} \frac{\theta}{2}} & 0 \\
0 & \mathrm{e}^{-\mathrm{i} \frac{\theta}{2}}
\end{array}\right),
$$

around $Z$ axis. For this reason, in order to design an architecture able to implement any single-qubit transformation (in turn, needed for a universal set of quantum gates), the proposed platform based on ESs must be able to realize two of the above transformations. In particular, the generic transformation $\mathbf{U}$ will be obtained using only $\mathbf{R}_{\mathbf{y}}$ and $\mathbf{R}_{\mathbf{z}}$, according to

$$
\mathbf{U}(\alpha, \beta, \theta)=\mathbf{R}_{\mathbf{z}}(\alpha) \mathbf{R}_{\mathbf{y}}(\theta) \mathbf{R}_{\mathbf{z}}(\beta) .
$$

In order to complete a universal set of quantum gates, a two-qubit transformation able to entangle qubits is necessary. We consider a two-qubit conditional phase shifter. This gate adds a phase factor $\mathrm{e}^{\mathrm{i} \gamma}$ to a given component of the two-qubit state, namely, to the state $|11\rangle$ :

$$
\begin{aligned}
& c_{a}|00\rangle+c_{b}|01\rangle+c_{c}|10\rangle+c_{d}|11\rangle \\
& \quad \stackrel{\mathbf{T}}{\longrightarrow} c_{a}|00\rangle+c_{b}|01\rangle+c_{c}|10\rangle+\mathrm{e}^{\mathrm{i} \gamma} c_{d}|11\rangle .
\end{aligned}
$$

For any value of the angle $\gamma$, not equal to an integer multiple of $\pi$, we have a transformation that, together with the onequbit gates $\mathbf{U}$, form a universal set.

On the lexicographically ordered basis $(|00\rangle,|01\rangle,|10\rangle,|11\rangle)$, the matrix representation of the conditional phase shifter is:

$$
\mathbf{T}(\gamma)=\left(\begin{array}{cccc}
1 & 0 & 0 & 0 \\
0 & 1 & 0 & 0 \\
0 & 0 & 1 & 0 \\
0 & 0 & 0 & \mathrm{e}^{\mathrm{i} \gamma}
\end{array}\right) .
$$

The transformation $\mathbf{T}$, acting on the two-qubit state, creates quantum entanglement between the two qubits, leading in general to a non-separable state. However, $\mathbf{T}$ does not change the relative weight of the four states. Thus, in order to detect the quantum entanglement, that is the basic resource for quantum computation, a composite transformation must be implemented, able to alter the detection probability of the states and, eventually, to create maximally entangled Bell's states, as, for example, a 'controlled NOT' [28].

A quantitative estimation of quantum entanglement generated by quantum gates can be obtained by computing the entanglement entropy. Indeed, while the dynamical properties of multi-partite entanglement [34], possibly affected by noise and a magnetic field [35-40], are still debated in literature, the bipartite entanglement of a pure state can be univocally assessed [41-43]. Considering the density matrix $\rho$ of the pure bipartite state, with subsystems $A$ and $B$, the entanglement $S$ is obtained as the von Neumann entropy of either $\rho_{A}$ or $\rho_{B}$, with $\rho_{A}\left(\rho_{B}\right)$ being the reduced density matrix of the subsystem $A(B): S=-\operatorname{Tr}\left(\rho_{A} \log \rho_{A}\right)$, where $\operatorname{Tr}$ is the trace operation.

\section{Charge transport in quantum-Hall ESs}

\subsection{ESs of a two-dimensional electron gas (2DEG) hosted by a semiconductor quantum well}

Non-interacting electrons with effective mass $m^{*}$ and charge $-e$ subject to a magnetic field $\mathbf{B}$ are described by the Hamiltonian $(\mathbf{p}+e \mathbf{A})^{2} /\left(2 m^{*}\right)$, where $\mathbf{p}$ is the momentum and $\mathbf{A}$ is the magnetic vector potential. If they are confined in a narrow semiconductor quantum well, they form a 2DEG whose dynamics in the direction perpendicular to the quantum well can be neglected in the limit of large energy level spacing of the well. Thus, the dynamics is two-dimensional, in a $x y$ plane, and considering $\mathbf{B}=(0,0, B)$ applied along the $z$ direction, the single-electron eigenstates are the well-known LLs with eigenenergies $\epsilon_{n}=\left(n+\frac{1}{2}\right) \hbar\left|\omega_{c}\right|$, where $\omega_{c}=-e B / m^{*}$ is the cyclotron frequency and $n=0,1,2, \ldots$ The above system is used to expose the quantum Hall effect [44], possibly the most celebrated consequence of magnetic quantization.

To derive the concept of Hall ESs and to set some definitions needed in the following sections, we follow the approach presented in [45]. Let us add an external confining potential $V_{\text {ext }}(x)$ to the 2DEG system, function of the $x$ coordinate alone, and choose the Landau gauge with magnetic vector potential $\mathbf{A}=(0, B x, 0)$, where $B$ is the constant modulus of the magnetic field. The Hamiltonian reads:

$$
\begin{aligned}
\hat{H}= & \frac{1}{2 m^{*}}(-\mathrm{i} \hbar \nabla+e \mathbf{A})^{2}+V_{\text {ext }}(x) \\
= & \frac{1}{2 m^{*}}\left(-\hbar^{2} \frac{\partial^{2}}{\partial x^{2}}+\left(-\hbar^{2} \frac{\partial^{2}}{\partial y^{2}}-2 \mathrm{i} \hbar e B x \frac{\partial}{\partial y}\right)\right. \\
& \left.+e^{2} B^{2} x^{2}\right)+V_{\text {ext }}(x) .
\end{aligned}
$$

Since the operator $\hat{P}_{y}=-\mathrm{i} \hbar \partial_{y}$ commutes with $\hat{H}$, the corresponding eigenstates with wave vector $k$ (eigenvalue of $\hat{P}_{y}$ ) and Landau index $n$ can be written in the form

$$
\Psi_{n, k}(x, y)=\varphi_{n}(x, k) \mathrm{e}^{\mathrm{i} k y} .
$$

They correspond to Landau states if $V_{\text {ext }}(x)$ vanishes. The 

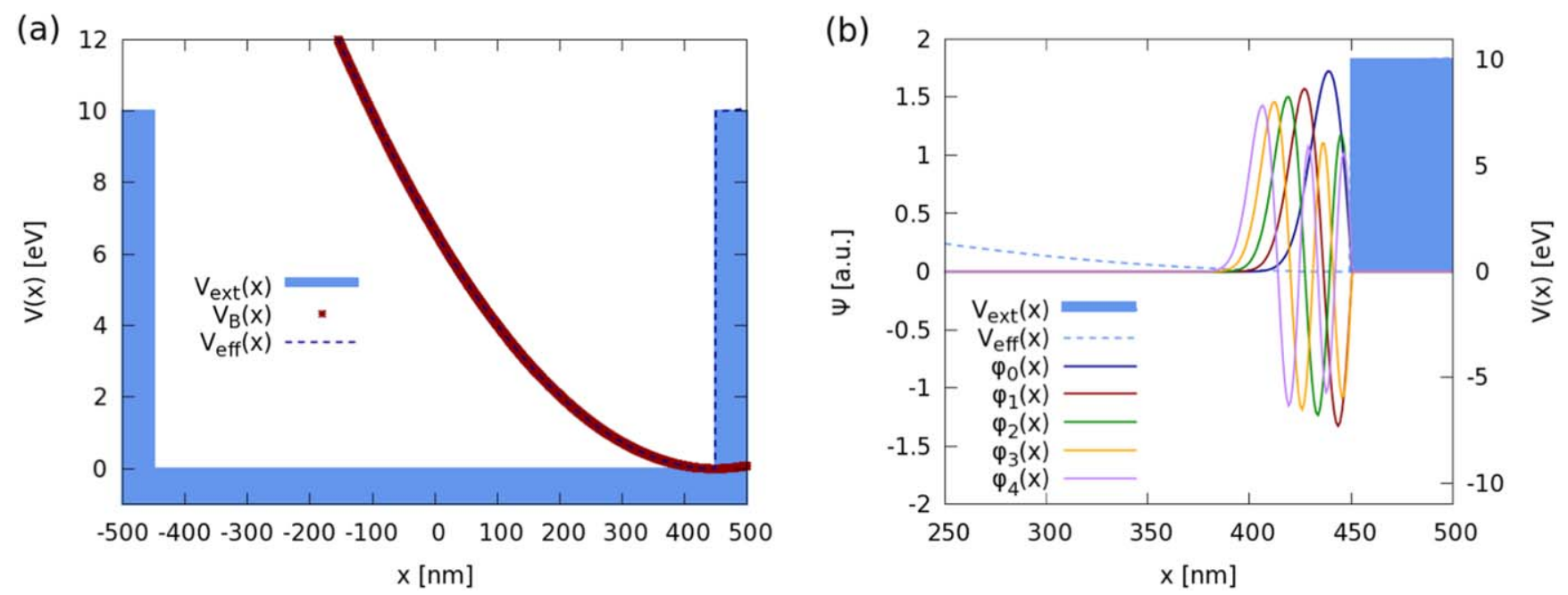

Figure 1. (a) Effective potential $V_{\text {eff }}(x)$ and its two components, namely the two last terms of equation (9), for an ES with $x_{0}=450 \mathrm{~nm}$ and for the confining potential described in the text. The total potential $V_{\text {eff }}$ corresponds to the magnetic term in the regions far from the potential barriers, while near the barriers it changes abruptly due to $V_{\text {ext }}$ (b) First five ESs $\varphi_{n}(x, k)$ in the potential $V_{\text {eff }}(x)$ at the right edge.

plane wave part $\mathrm{e}^{\mathrm{i} k y}$ corresponds to a free motion along the $\hat{y}$ direction, while the transverse component $\varphi_{n}(x, k)$, the so called ES, is an eigenfunction of the 1D effective Hamiltonian:

$$
\hat{H}^{\mathrm{eff}}(k)=\frac{-\hbar^{2}}{2 m^{*}} \frac{\partial^{2}}{\partial x^{2}}+\frac{1}{2} m^{*} \omega_{c}^{2}\left(x+\frac{\hbar}{e B} k\right)^{2}+V_{\mathrm{ext}}(x) .
$$

The last two terms of $\hat{H}^{\text {eff }}$ represent an effective potential

$$
V_{\mathrm{eff}}(x)=\frac{1}{2} m^{*} \omega_{c}^{2}\left(x-x_{0}\right)^{2}+V_{\mathrm{ext}}(x),
$$

where

$$
x_{0}(k)=-\frac{\hbar k}{e B}
$$

is the center of the magnetic parabolic confinement along $\hat{x}$. If $V_{\text {ext }}(x) \neq 0$, the energy bands, i.e. the perturbed LLs, eigenvalues of $\hat{H}^{\text {eff }}(k)$, depend explicitly on $k$ :

$$
E_{n}(k)=\hbar\left|\omega_{c}\right|\left(n+\frac{1}{2}\right)+\epsilon_{n}(k),
$$

or, from a different perspective, on the position $x_{0}$ according equation (11). In the last expression, $\epsilon_{n}(k)$ is a positive correction depending on the specific form of $V_{\text {ext }}(x)$, and gives the dispersion relation of the bands.

Typically, for electrons of a 2DEG nosedives, the external potential $V_{\text {ext }}(x)$ is flat in the central region, while it defines a barrier near the edges of the device itself. The main effect of this confining potential is the bending upwards of the energy bands at the edges. As we will see, this induces a net group velocity for localized states lying at the border of the device.

Figure 1(a) displays a simple square confining potential $V_{\text {ext }}(x)$ together with the parabolic term of equation (10) and the total $V_{\text {eff }}(x)$. The ensuing five ESs with lower energy, localized on the right side of the domain, are shown in figure 1(b). These states constitute chiral channels that allow for coherent electronic transport in the $y$ direction. However, it should be noticed that for carriers localized far from the barrier, the effects of the external potential is negligible. Therefore, the solutions of the effective Schrödinger equation are still LLs, eigenstates of the harmonic oscillator, as the correction $\epsilon_{n}(k)$ of equation (12) is null.

Specifically, the confining potential of figure 1 and figure 2 is $V_{\text {ext }}(x)=V_{\max }[\Theta(-x-L)+\Theta(x-L)]$, and describes a device width $\Delta x=2 L$ where the confining barrier has height $V_{\max }$ (with $L=450 \mathrm{~nm}$ and $V_{\max }=10 \mathrm{eV}$ ) and $\Theta$ is the Heaviside step function.

As stated above, the general effect of the barrier is to bend upwards the bands in the adjacent regions of space, as shown in figure 2 . Then, as $k$ (corresponding to the 'center' $x_{0}$ of the wave function) approaches the position of the barrier, the energy of the eigenfunction increases, and its form $\varphi_{n}(x$, $k$ ) along $\hat{x}$ loses its symmetry with respect to $x_{0}$. The center $x_{0}$, which is still defined by equation (11), can be even deeply inside the barrier, while the wave function remains mainly outside it and it may be quite different from the harmonic oscillator one. $x_{0}$ is no more the center of symmetry of the eigenstate, but it is still a good quantum number and the center of the magnetic parabolic potential in equation (10). We can therefore express the bands $E_{n}(k)$ also in terms of $x_{0}$ through the inverse of equation (11). Concerning this point, we must stress that the wavevector $k$ appearing in equation (8) is not a physical observable (it is not the true wavevector of the electron), since it depends, through $x_{0}$, from the origin of the coordinate system. Therefore, it is a gauge-dependent quantity. For the system under study, this point will be explicitly dealt with in section 4.2.

From an analogy between the quantum and classical expressions for energy

$$
\hbar\left|\omega_{c}\right|\left(n+\frac{1}{2}\right)=\frac{1}{2} m \omega_{c}^{2} r^{2}
$$

we can obtain a relationship between the radii of quantum and classical orbits, namely $l_{m} \sqrt{2 n+1}=r_{n}$, where 

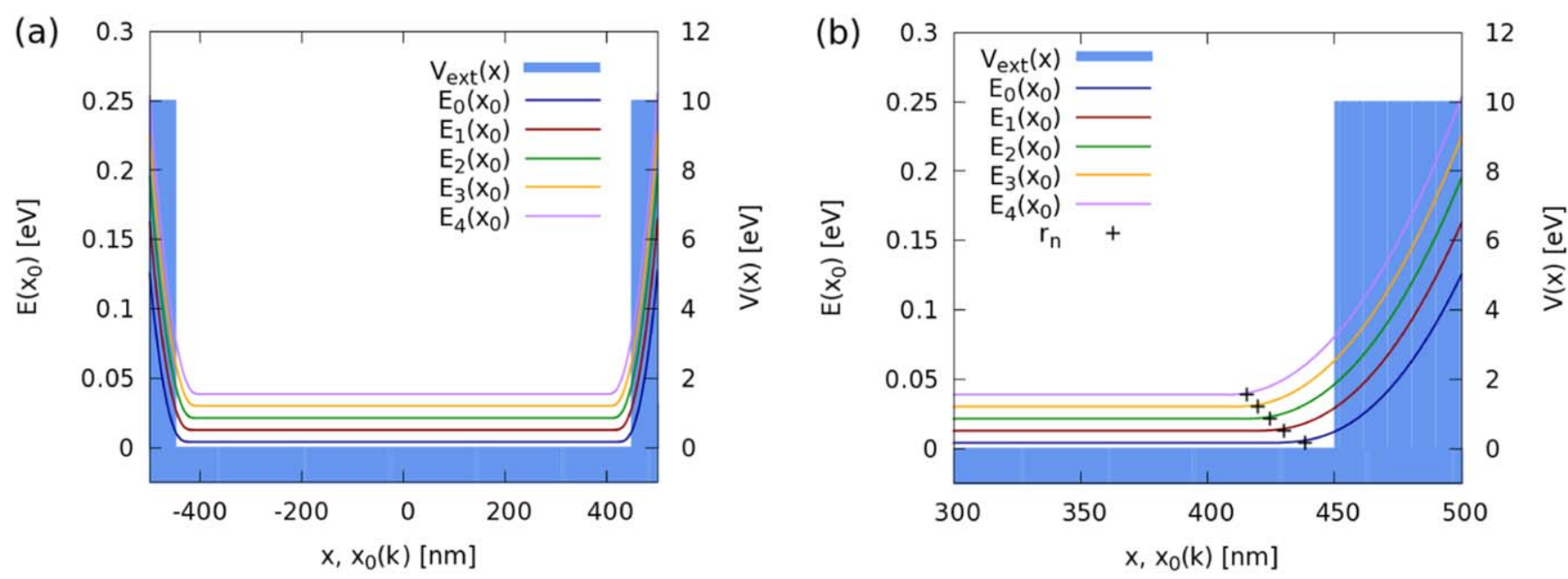

Figure 2. (a) Band structure $E_{n}\left(x_{0}(k)\right)$ for the first five LLs, calculated numerically, in presence of the square external potential profile $V_{\text {ext }}(x)$ (light blue shaded area) at $B=5 \mathrm{~T}$. Energies are displayed as a function of the parameter $x_{0}(k)$ by using equation (11). (b) Band structure $E_{n}\left(x_{0}(k)\right)$.

$l_{m}=\sqrt{\hbar / e B}$ is the magnetic length. Each eigenstate of energy $E_{n}$ has a corresponding 'classical radius' $r_{n}$, which is discretized in multiples of the magnetic length. This parameter expresses the spatial extension of the eigenstate along the $x$ direction, for any value of the quantum number $n$ $[45,46]$. Indeed, even if the eigenfunctions show a common harmonic confinement around $x_{0}$, the presence of the Hermitepolynomials changes the width of $\varphi_{n}(x, k)$ with respect to that of a simple Gaussian. This parameter can be also interpreted as a measure of the space needed by the carrier to behave as a free particle, only subject to the magnetic field. Indeed, as we can see in the band representation of figure 2(b), the $n$th LL band starts to bend when near the edge: for every band of quantum number $n$, the black cross indicates the distance $r_{n}$ from the edge of the barrier at an energy of the unperturbed LL (i.e. the value of $E_{n}$ in the flat region). the center of the state $x_{0}$ is at a distance of about $r_{n}$ from the barrier, thus changing its behavior from a Landau state to an ES. Therefore, particles of higher energy can be perturbed by an external potential even at larger distances from the confining barrier.

It is important to stress the chiral nature of charge transport inside the edge channels, which is due to the magnetic field: chirality means that the charge transport inside the channel can only occur in a fixed direction-which is reversed at the two opposite edges of the device. As it happens for skipping orbits, the edge channels are therefore immune to back-scattering, so they represent ideal candidates for the coherent ballistic transport required in quantum computing devices.

\subsection{Carrier injection in ESs: injection of single localized electrons or a current of delocalized electrons}

Thanks to the properties described above, ESs are exploited to quench decoherence phenomena [47] in electron transport in semiconductor-based devices. In real systems, the LLs always produce ESs, due to the finite dimensions of the devices. As already stated in the previous section, well inside the samples the bands are flat, while at the borders they bend, typically intersecting the Fermi level (see figure 3). The number of LLs that cross the Fermi energy gives the number of ESs available for conduction at the edge of the device. Due to the linear relation between the cyclotron frequency $\omega_{\mathrm{c}}$ and the magnetic field in equation (12), an increase of the magnetic field pushes the LLs towards higher energies, and the last occupied LL can eventually be depleted. Furthermore, a narrow constriction in the 2DEG can put in contact two ESs of two opposite edges, thus allowing the electrons to be reflected in the opposite $y$ direction.

Electron devices operating in the IQH regime are usually fabricated in a high mobility 2DEG embedded in a semiconductor heterojunction (e.g. GaAs-AlGaAs), with Ohmic contacts connected to the edges of the structure as source/ drain leads. However, the presence of ESs and the possibility of engineering their path is not limited to semiconductor systems [48]. The strong perpendicular magnetic field originates a new correlated state that behaves as an incompressible liquid with a fixed and well-defined density $n$ [3]. In the IQH regime, this is generally related to the bulk $F F \nu=\frac{n h c}{e B}$, that is the number of LLs that are filled in the bulk of the confined 2DEG. This parameter is controlled by the depletion of the 2DEG with metallic gates on top/bottom of the heterostructure, and it can be varied locally with modulation gates to control the path of the ESs in the internal region.

As anticipated above, ESs are ideal candidates to implement semiconductor logic gates for flying-qubit quantum computing architectures, due to their large coherence length. In fact, single-electron interferometry can in principle realize single-qubit transformations, while the mutual Coulomb interaction of propagating carriers can couple different qubits.

Indeed, the first experimental implementations of quantum ES interferometry were based on a continuous stream of electrons injected in an available ES of the 2DEG through an Ohmic contact [8, 11, 49-52]. In these implementations, the 


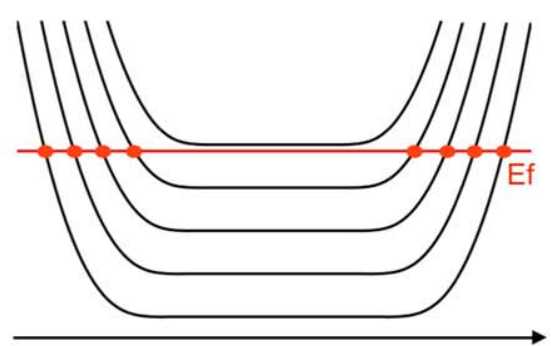

$B=4 T$

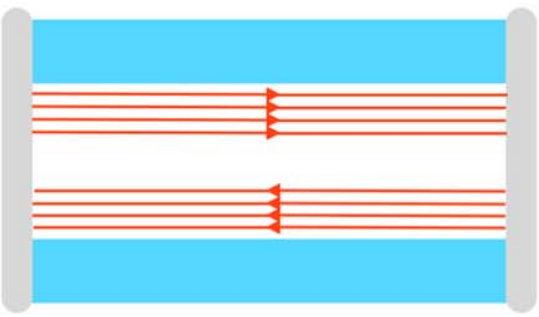

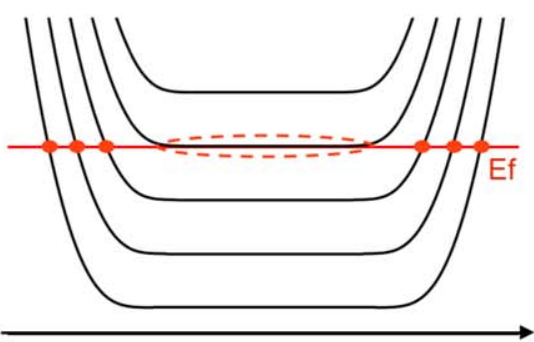

$B=5 T$

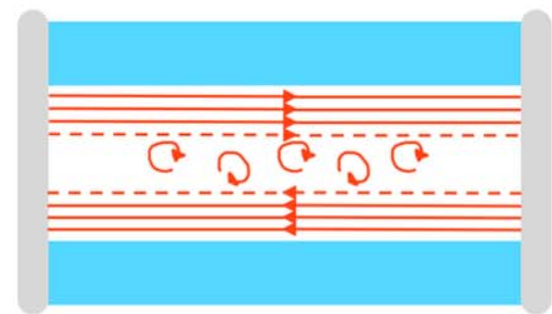

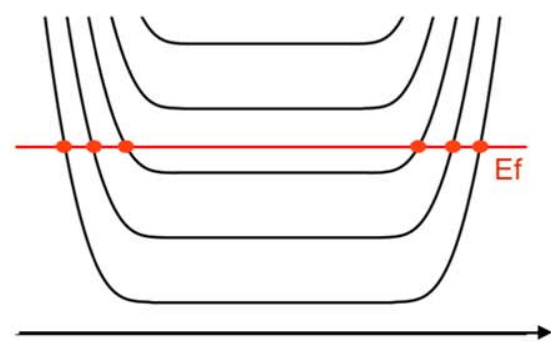

$B=6 T$

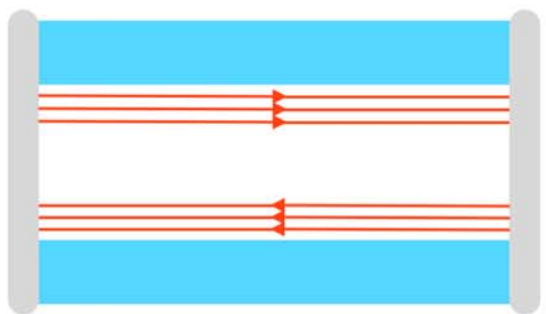

Figure 3. (Top) Band structure in real space of the device for the LLs at different values of $B$. (Bottom) Evolution of the edge channel paths with increasing magnetic field.

ES wave function is localized only along the transverse direction of the device, so that the interference is realized between currents. The quantum properties of the system are assessed via ensemble measurements of noise and the detection of the transmitted current [49, 50]. These interfering currents are usually modeled in a time-independent framework by means of scattering states [53-56]. In this picture, each component of the logic gate can be represented by a scattering matrix with properly defined reflection and transmission coefficients. The many-body electron state representing the flow of electrons from the leads is built by applying creation and annihilation operators to the ground state (a filled Fermi sea with negative energies). The zero frequency cross correlation of the current fluctuations is generally related to the single-particle detection probability in a given output of the device, or to the joint detection probability in two different outputs, which is experimentally available by means of ensemble measurements. This framework has been applied to study how Coulomb interaction between an interferometer and a detector can be exploited to generate entanglement and which-path detection [54], to predict the violation of Bell's inequality in the HanburyBrown-Twiss (HBT) interferometer [53] or unexpected increase of the visibility at large biases in a MZI [55].

Only recently, experiments have focused on the interference of single localized carriers [57-60]. From a quantuminformation processing perspective, the possible encoding of qubits is simpler than in the previous scenario, as the qubit state can be represented by the spin state, the Landau index $n$, or the spatial localization of the carrier. In the following sections we will focus on the modeling of quantum logic gates with the two latter qubit encodings.

Different protocols for the injection of electrons in WPs of ESs are nowadays available, but single-electron detection is still a challenging task due to the high velocity of the WPs and short interaction time with the detector [61]. Within this framework, thanks to the quasi-parabolic dispersion of a single ES, transport in edge channels is usually modeled in a 1D approach by means of WPs of plane waves [62-65] or in a full 2D approach with time-dependent solvers [66-69]. In the following section, we will sketch the three available protocols for the injection of single carriers in ESs.

\subsection{Quasi-particle wave function of electrons propagating in ESs: Levitons, Lorentzian and Gaussian wave functions}

The on-demand generation of pure quantum excitations is crucial for the controlled propagation of single carriers in ESs, but it is particularly difficult for a system of fermions where an external voltage bias affects the states below the Fermi sea, thus resulting in a complex superposition of electrons and holes.

A protocol to inject single-electrons in edge channels was first proposed by Levitov [9, 70, 71], who suggested that it is possible to generate a minimal excitation, later named 'Leviton', containing only one electron charge and no holes, with a properly time-dependent perturbation $V(t)$ of Lorentzian shape:

$$
V(t)=-\frac{\hbar}{e} \frac{2 \tau}{\left(t-t_{0}\right)^{2}+\tau^{2}},
$$

where $\tau$ is the emission time and $t_{0}$ the instant of maximum voltage (a sort of 'emission time' for the WP describing the electron). As a consequence of the modulated bias, the Fermisea re-organizes itself and a single electron (with no accompanying hole) is emitted in the conduction band. The normalized electronic wave function in the energy domain is given by [63]:

$$
\Phi(E)=\sqrt{2 \tau} \mathrm{e}^{-\left(\tau-\mathrm{i} t_{0}\right)\left(E-E_{\mathrm{F}}\right)} \Theta\left(E-E_{\mathrm{F}}\right),
$$

with $E_{\mathrm{F}}$ Fermi energy of the system. In general, the Leviton 
wave function is given by the Fourier transform of equation (15), once the energy dispersion relation $E=E(k)$ is known:

$$
\begin{aligned}
\psi(x, t) & =\frac{1}{\sqrt{2 \pi}} \int \mathrm{d} k \mathrm{e}^{\mathrm{i} k x} \tilde{\psi}(k) \mathrm{e}^{-\frac{\mathrm{i}}{\hbar} E(k)\left(t-t_{0}\right)} \\
& =\frac{1}{\sqrt{2 \pi}} \int \mathrm{d} k \mathrm{e}^{\mathrm{i} k x}\left[\Phi(E(k))\left|\frac{\mathrm{d} E}{\mathrm{~d} k}\right|\right] \mathrm{e}^{-\frac{\mathrm{i}}{\hbar} E(k)\left(t-t_{0}\right)} .
\end{aligned}
$$

However, usually this equation can not be treated analytically and further approximations are needed. For a 1D system (in our case the $y$ axis) with a linear dispersion, i.e. $E(k)=v \cdot k$, the Leviton wave function is given by a Lorentzian function:

$$
\psi(x, t)=\frac{1}{\sqrt{\pi}} \frac{\sqrt{\frac{\Gamma}{2}}}{\left(x-x_{0}\right)-v\left(t-t_{0}\right)+\mathrm{i} \frac{\Gamma}{2}},
$$

where $\Gamma=2 v \tau$ is the full width at the middle height of the density probability $|\psi(x, t)|^{2}$. A major advantage of Levitons, in comparison to alternative injection protocols, comes from their minimal excitation character. In fact, a Leviton injected at the Fermi energy, is less affected by energy relaxation processes [72]. We also note that the generation of Levitons relies on a properly designed voltage pulse on the source lead and does not require additional nanolithography of the sample. The generation of a minimal excitation containing a single electron charge has been designed theoretically and proven experimentally in Hong-Ou-Mandel (HOM) and HBT interferometers $[60,73]$ : the WPs are partitioned at a QPC and shot noise measurements reveal the number of electron and holes in the beam. This number is measured to be equal to a single electron charge for Lorentzian pulses, contrary to alternative shapes of the periodic drive, which proves the absence of additional holes propagating in the device.

An alternative method to inject single electrons is realized by means of a capacitor, as a dynamic quantum dot, weakly connected to the lead where a single charge is injected or absorbed [74]. The implementation of this protocol in the IQH regime was originally proposed by Büttiker in [75]: a quantum dot, connected to a 2DEG, is initially in a Coulomb blockade regime with the same Fermi energy inside and outside. A periodic voltage bias is then applied to the dot to shift the last occupied level above the Fermi energy inside the 2DEG, so that a single-electron, also called 'Landau quasiparticle', is injected into the device. In the next semi-period, the level drops below the Fermi energy and collects an electron from the 2DEG, corresponding to the injection of a hole. Consequently, no DC current can be produced by this source, but only AC current of alternated electrons and holes. These mesoscopic capacitors are realized in the IQH regime by means a tunable QPC that controls the tunnel-coupling with the 2DEG [57]. Due to the presence of the perpendicular magnetic field, ESs run along the boundary of the confined region and generate the discrete levels in the dot. By defining $\tau_{e}$ the emission time of the electron from the mesoscopic capacitor and $\epsilon_{0}$ its energy, the electronic WP in 1D for a linear dispersion [24]

$$
\psi\left(t ; \epsilon_{0}, t_{0}\right)=\frac{\Theta\left(t-t_{0}\right)}{\sqrt{\tau_{\mathrm{e}}}} \mathrm{e}^{-\mathrm{i} \epsilon_{0}\left(t-t_{0}\right) / \hbar} \mathrm{e}^{-\left(t-t_{0}\right) /\left(2 \tau_{\mathrm{e}}\right)}
$$

can be rewritten as a Lorentzian pulse in energy:

$$
\tilde{\psi}\left(\epsilon ; \epsilon_{0}, t_{0}\right)=\frac{N \mathrm{e}^{\mathrm{i} \epsilon t_{0} / \hbar}}{\left(\epsilon-\epsilon_{0}\right)+\mathrm{i} \frac{\Gamma}{2}},
$$

with $\Gamma=\hbar / \tau_{e}$ and $N$ the normalization constant. The quality of this single electron source is assessed by an ensemble measurement of the high-frequency current autocorrelation function, which shows the existence of a fundamental noise, called quantum jitter, entirely determined by the quantum uncertainty on the emission time of a single charge [10]. However, mesoscopic capacitors are more affected by interchannel and intra-channel interactions in presence of a double FF [72] with respect to Lorentzian pulses. Levitons are indeed quasiclassical charge density waves that does not entangle with the environment because the interaction region acts as a frequency-dependent BS. On the contrary, the Landau quasiparticles are superpositions of such density waves, so that entanglement with the second edge channel can destroy their coherence. Inter-channel interactions at FF 2 produce therefore charge fractionalization, affecting the bunching probability of an electronic HOM interferometer [12, 24, 76-79]. Because of this source of decoherence, the minimum of the bunching probability, also known as Pauli dip, does not vanish for a fermionic two-particle system, but rather depends on the emission time of the Landau quasi-particle [80, 81]. A numerical simulation aimed at revealing the Pauli dip in a HOM interferometer will be presented in section 6.1.

The decoherence processes induced by inter-channel and intra-channel interactions can be quenched by injecting single electrons at energies much larger than the Fermi sea, as done by quantum dot pumps with tunable barriers [82]. The singleelectron injection is here realized by means of a quantum dot coupled to the source region and to the device by timedependent confining potentials. One of the two potential barriers is lowered below the Fermi sea of the electronic reservoir and then it is progressively raised in order to trap one or a small number of electrons in the confined region. The same potential barrier is further increased, so that when it exceeds the other confining barrier, the trapped electrons are injected in the 2DEG with an energy well above the Fermi energy of the 2DEG [83]. Ryu et al also proposed a theoretical protocol to generate and detect almost identical Gaussian WPs from non-identical quantum dot pumps [84]. Recently, the modeling of on-demand electron sources also addresses the specific energy distribution of the emitted carriers [85, 86], and theoretical studies [87] analyze the magnetic and energetic regime to quench decoherence and relaxation processes induced by inelastic scattering with acoustic and optical phonons.

In the numerical simulations described in the following sections, the choice of the initial wave function of the propagating carrier is arbitrary. Except for specific examples aimed at assessing the impact of the initial electron energy 
distribution on the transmission characteristics of the system, we consider a minimum uncertainty WP as the one that is generated by the protocol described in [84]. Specifically, the single carrier is initialized in the wave function

$$
\psi(x, y)=\frac{1}{\sqrt{2 \pi}} \sum_{n} \int \mathrm{d} k F_{n}(k) \mathrm{e}^{\mathrm{i} k y} \phi_{n}(x, k),
$$

that corresponds to a linear combination of ESs $\varphi_{n}(x, k)$ with the Gaussian weight

$$
F_{n}(k)=\sqrt[4]{\frac{2 \sigma^{2}}{\pi}} \mathrm{e}^{-\sigma^{2}\left(k-k_{0}\right)^{2}} \mathrm{e}^{-\mathrm{i} k y_{0}},
$$

where $k_{0}$ is the central wave vector of the carrier and $\sigma$ is the real-space broadening of the WP. The variance in $k$-space $\sigma_{k}$ is computed from $\sigma_{k}^{2}=\frac{1}{4 \sigma^{2}}$. Note that, differently from a delocalized ES, the WP in equation (20) can be normalized, so that its squared modulus $|\Psi(x, y)|^{2}$ is directly interpreted as the probability of finding the charge at the coordinate $(x, y)$. We usually assume that the electron is injected selectively in one channel $n$ and no superposition between different LLs is initially present, i.e. $F_{n}(k) \neq 0$ for a single value of $n$ at $t=0$. When the notation is not ambiguous, we will omit the index $n$ in the corresponding weight $F(k)$. The relation between the center of the ES $x_{0}$ and its wave vector $k$ (see equation (11)) implies that an ES significantly contributes to the dynamics of the WP only if:

$$
x_{0} \approx\left|x_{0}\left(k_{0}+3 \sigma_{k}\right)-x_{0}\left(k_{0}-3 \sigma_{k}\right)\right|=\frac{3 l_{m}^{2}}{\sigma} .
$$

The WPs are initialized in a region of the device where the external potential is translationally invariant with $y$. For a more realistic description of the confining potential defining the ESs, instead of taking a square confinement as in figure 2, we consider a smooth confinement modeled by means of a Fermi-like function:

$$
V_{\mathrm{ext}}(x)=\frac{V_{0}}{1+\exp \left(\frac{x-x_{b}}{\lambda}\right)},
$$

with $x_{b}$ and $\lambda$ defining the position and the smoothness of the barrier, respectively. Through equation (11), the definition of $x_{0}$ also fixes the central momentum of the WP and its velocity. Indeed, the weight function on the $k$-space $F(k)$ can also be rewritten in terms of $x_{0}(k)$, so that the energy dispersion of the WP is related to the profile of the initialization potential, as depicted in figure 4.

By assuming $x_{0}$ sufficiently far from the confining potential, we can provide an analytical expression of the ESs $\varphi_{n}(x, k)$, and equation (20) can be integrated, leading to:

$$
\Psi(x, y)=\frac{1}{\pi} \frac{\sqrt[4]{\frac{2 \sigma^{2}}{l_{m}^{2}}}}{\sqrt{2 \sigma^{2}+l_{m}^{2}}} \mathrm{e}^{-k_{0} \sigma^{2}} \mathrm{e}^{-\frac{x^{2}}{2 l_{m}^{2}}} \mathrm{e}^{\frac{\left(\operatorname{sgn}(B) x-\mathrm{i}\left(y-y_{0}\right)-2 k_{0} \sigma^{2}\right)^{2}}{2\left(2 \sigma^{2}+l_{m}^{2}\right)}} .
$$

Here, $|\Psi(x, y)|^{2}$ describes a localized 2D-Gaussian probability distribution in space, with anisotropic standard deviations

$$
\sigma_{x}^{2}=\frac{l_{m}^{2}}{2}\left(1+\frac{l_{m}^{2}}{2 \sigma^{2}}\right)
$$

$$
\sigma_{y}^{2}=\sigma^{2}\left(1+\frac{l_{m}^{2}}{2 \sigma^{2}}\right)
$$

This analytical expression is valid only for states that are degenerate in energy, i.e. inside the bulk of the 2DEG, which do not contribute to transport. When $x_{0}$ approaches the confining barrier at the edge, the eigenstates must be computed numerically, and the initial wave function is built by summing over the relevant components of the WP according to equation (20).

The electron wave function in equation (20) and the quasi-parabolic dispersion of the combined ESs in figure 4(b) highlight the similarities between a WP of ESs and a WP of plane waves. This analogy is usually exploited to study electron transport in the IQH regime by means of 1D analytical models, as also described in our theoretical investigation of the multichannel MZI [69] and the two-particle HOM interferometer [88]. Within this simplified model, the quantum logic gates are described by a product of scattering matrices and the probability of detection or joint detection at the drains are obtained by integrating over the energy dispersion of the final WP. However, such analytical 1D model requires a number of approximations on the energy dependence of the transmission coefficient and does not include the exact Coulomb repulsion for interacting particles. Additionally, electron-electron interactions in a 2D scenario generally differ from the 1D case [89], as proved by the computation of bunching probability of two strongly-interacting particles, later described in section 6.1. The exact simulation of singleparticle or two-particle transport in WPs of ESs requires therefore alternative approaches, able to describe exactly the potential background experienced by the carriers. To this aim, we implemented in-house a numerical solver of the timedependent Schrödinger equation for a single-particle and a two-particle WP in 2D. Here, we properly design the potential background $V_{\text {ext }}(x, y)$ of the travelling WP, in order to mimic the effect of top gates in a realistic electron interferometer, as described in the following sections. By resorting to the FFTW3 library and LAPACK routines, the wave function is evolved with the Split-Step Fourier Method, based on the iterative application of a properly defined evolution operator. A two-particle version of the simulator, parallelized in the MPI paradigm to overcome memory occupation problems, has also been developed to study two-particle correlations in Hall nanointerferometers. Results will be presented in sections 5 and 6 , together with a detailed description of the numerical approach in section 5.1. By using this exact model, we are able to assess the dynamic properties of the propagating WP and easily include electron-electron interaction or time-dependent potentials in the simulations. As an example, the exact inclusion of time-dependent potentials finds an important application in the numerical simulation of electrontransport sustained by surface acoustic waves [90-93], which is another highly efficient on-demand protocol to control single-electrons transport in semiconductors [94]. 
(a)

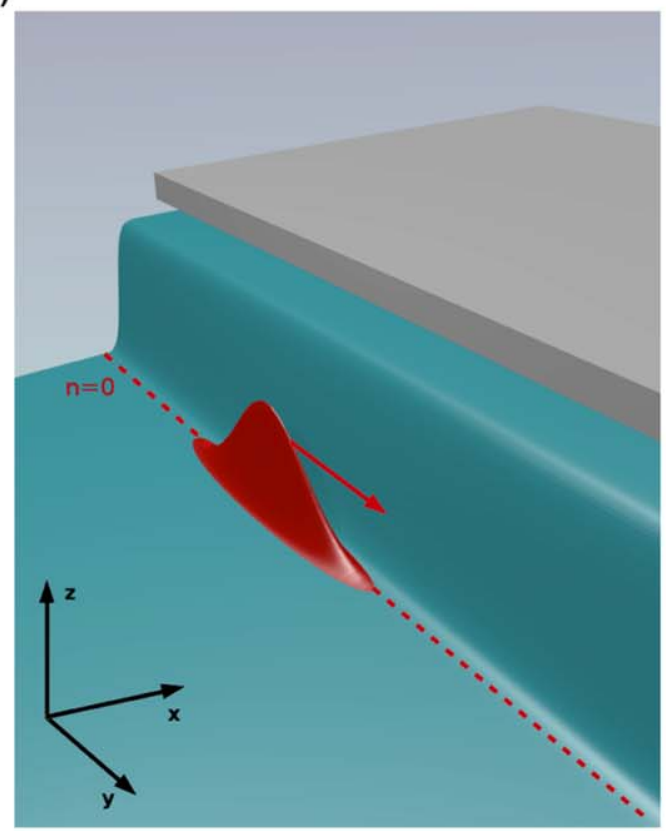

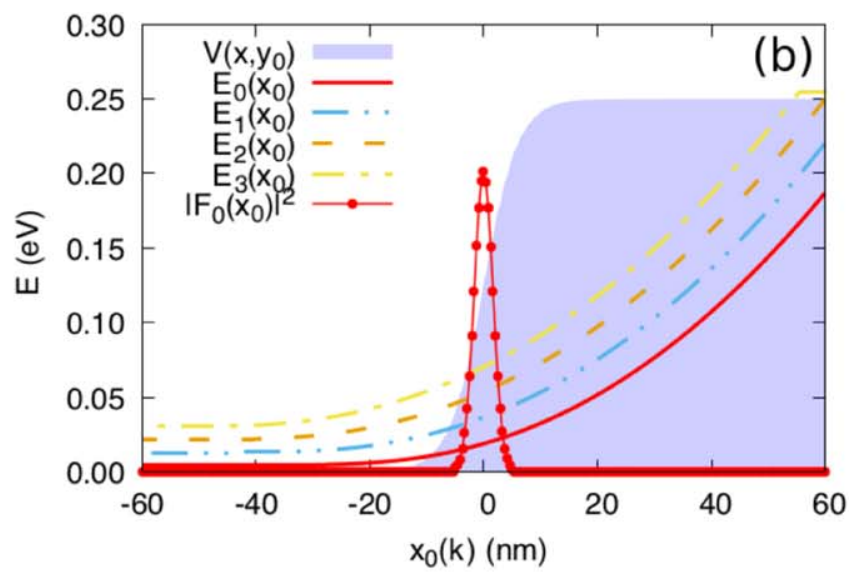

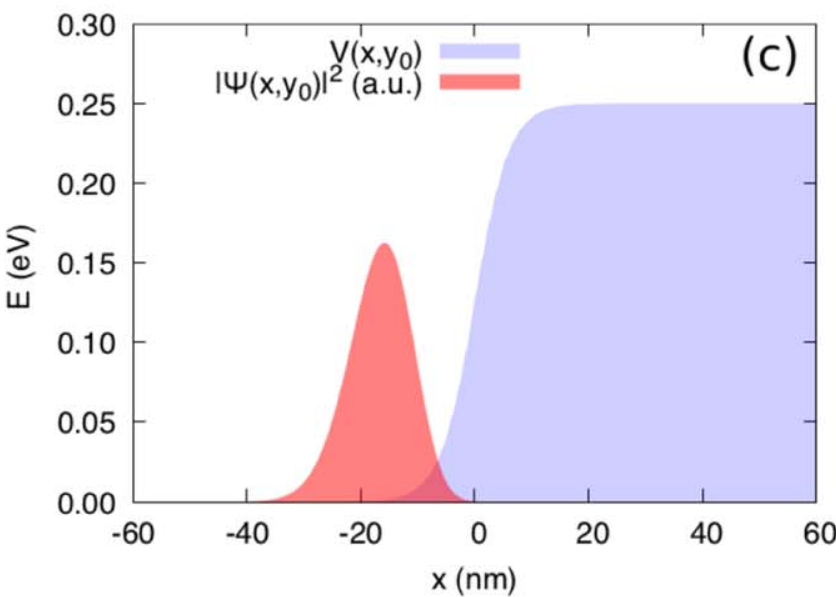

Figure 4. Linear combination of ESs according to equations (20)-(22) (here $\sigma=40 \mathrm{~nm}, y_{0}=+550 \mathrm{~nm}$ and $k_{0}$ is calculated from $x_{0}=0 \mathrm{~nm}$, corresponding to the edge of the barrier). (a) 3D rendering of $\Psi(x, y)$ (red shape) in the confining external potential (blue surface) generated by a top gate (gray area). (b) Composition of the wave function in terms of $F_{n}(k)$, together with band dispersion relation for the first 5 bands and the external potential. (c) Real-space representation of the wave function on the $x$ axis for a fixed $y$ corresponding to the WP maximum. The smoothed external potential $V_{\text {ext }}(x)$ is also reported.

\section{Controlling and mixing ESs}

A flying-qubit implementation of a quantum computing architecture requires the capability of manipulating ESs to reproduce logic gate operations. In the following, two geometries will be addressed, both operating in the IQH regime, with two alternative definitions of the two qubit states $|0\rangle$ and $|1\rangle$. At bulk FF 1 , where only the first LL $n=0$ is energetically available, the $|0\rangle$ state is represented by the localization of the propagating carrier in the $\operatorname{ES} \varphi_{0, k}(x)$, while the $|1\rangle$ state corresponds to the counterpropagating one, $\varphi_{0,-k}(x)$, with opposite chirality. At bulk FF 2, the basis states are the copropagating energy-degenerate ESs with different Landau indexes (but the same chirality): $\varphi_{0, k}(x)$ for $|0\rangle$ and $\varphi_{1, k}(x)$ for $|1\rangle$. In the following of this work, we present the modeling of the main building blocks for single-qubit and two-qubit logic operations in both scenarios.

\subsection{Carrier dispersion and velocity}

Thanks to their chirality and parabolic dispersion, electrons in ESs $\varphi_{n, k}$ are usually mapped to free particles in a 1D free space with a renormalized mass. This analogy holds also for
Gaussian WPs of ESs (see section 3.3). For an analytical demonstration, (i) a unitary bulk FF and (ii) a limited number of ESs combined in the WP, are assumed. The latter requirement is fulfilled for a small enough energy broadening $\left(\sigma_{k}=1 / \sigma\right)$ with respect to the energy scale of the first LL $E_{0}(k)$ around the center of the WP, $k_{0}$. As shown in figure 4, near the edge, $E_{0}(k)$ is well approximated by a parabolic function, $E_{0}(k)=a k^{2}+b k+c=a\left(k-k_{1}\right)^{2}+c^{\prime}$, with $a$, $b, c$, or $c^{\prime}, k_{1}$ fitting parameters depending on the transverse confining potential $V(x)$. The eigenstates are all almost identical, $\varphi_{1}(x, k) \sim \varphi_{1}\left(x, k_{0}\right)$, so that equation (24) is well approximated with:

$$
\Psi(x, y)=\frac{\mathrm{e}^{-\frac{\left(y-y_{0}\right)^{2}}{4 \sigma^{2}}} \mathrm{e}^{\mathrm{i} k_{0} y}}{\sqrt[4]{2 \pi \sigma^{2}}} \varphi_{1}\left(x, k_{0}\right),
$$

which is factored along the two spatial axis. For a translationally invariant confining potential in the $\hat{y}$ direction, the $x$ component of the wave function is not modified by the evolution $\left(\varphi_{1}(x, k)\right.$ is an eigenstate of the effective 1D Hamiltonian in equation (9)), while along the $\hat{y}$ direction the electron state describes a Gaussian wave function [95] centered at $y_{0}$. The time-dependent wave function is computed by 
applying the evolution operator,

$$
\begin{aligned}
& \Psi(x, y ; t)=\mathrm{e}^{-\frac{\mathrm{i}}{\hbar} \hat{H}_{L} t} \Psi(x, y) \\
& =\frac{1}{\sqrt{2 \pi}} \int \mathrm{d} k \sqrt[4]{\frac{2 \sigma^{2}}{\pi}} \mathrm{e}^{-\sigma^{2}\left(k-k_{0}\right)^{2}} \mathrm{e}^{\mathrm{i} k\left(y-y_{0}\right)} \varphi_{1}\left(x, k_{0}\right) \mathrm{e}^{-\frac{\mathrm{i}}{\hbar} E_{1}(k) t} \\
& =\varphi_{1}\left(x, k_{0}\right) \frac{\mathrm{e}^{-\frac{\mathrm{i} c t}{\hbar}} \mathrm{e}^{-\frac{\left(y-y_{0}+2 i k_{0} \sigma^{2}+\frac{b t}{\hbar}\right)^{2}}{4\left(\mathrm{i} \frac{a}{\hbar} t+\sigma^{2}\right)}} \mathrm{e}^{-k_{0}^{2} \sigma^{2}}}{\sqrt[4]{\frac{2 \pi}{\sigma^{2}}} \sqrt{\sigma^{2}+\frac{\mathrm{i} a t}{\hbar}}}
\end{aligned}
$$

while the probability distribution in the $\hat{y}$ direction is a Gaussian with a time-dependent spreading related to the slope of $E_{0}(k)$ :

$$
\begin{aligned}
|\Psi(x, y ; t)|^{2} & =\left|\varphi_{1}\left(x, k_{0}\right)\right|^{2} \frac{\mathrm{e}^{-\frac{\left(y-y_{0}+\frac{2 a\left(k_{0}-k_{1}\right.}{\hbar} t\right)^{2}}{2\left(\frac{a^{2} t^{2}}{\hbar^{2} \sigma}+\sigma^{2}\right)}}}{\sqrt{2 \pi\left(\sigma^{2}+\frac{a^{2} t^{2}}{\hbar^{2} \sigma^{2}}\right)}}, \\
\sigma^{2}(t) & =\sigma^{2}+\frac{a^{2} t^{2}}{\sigma^{2} \hbar^{2}} .
\end{aligned}
$$

The probability distribution in equation (29) evolves as a Gaussian WP of plane waves with an effective wave vector $\Delta k=k_{0}-k_{1}$ and group velocity $v_{g}=\frac{2 a \Delta k}{\hbar}$, which clearly differs from the one in GaAs heterostructures. To restore the analogy with a Gaussian WP of plane waves, the group velocity is rewritten in terms of the gauge-independent wave vector $\Delta k$ and an effective magnetic mass $m_{B}=\frac{\hbar^{2}}{2 a}$. This renormalized mass incorporates the effects of the magnetic field on electron transport in ESs and depends on the band dispersion $E_{0}(k)$ trough its slope $a$. It should be noticed that the above considerations can be generalized to any LL, so that the group velocity differs for cyclotron-resolved ESs, i.e. $v_{g}^{n}=\frac{\hbar \Delta k}{m_{B}^{n}}$. Moreover, the confining potential of ES-based devices can also be designed to induce an almost linear dispersion of the LLs [69]. This reduces the spatial broadening of the electron WP during its time evolution, thus increasing its spatial control.

\subsection{Spatial control (phase shifter)}

The phase shifter $\mathbf{R}_{\mathbf{z}}$ already introduced in section 2 is a single-qubit gate that induces a phase difference between the two basis states. In the scattering matrix formalism, the transmission coefficients from channel $i$ to channel $j$ are therefore Dirac deltas in their module, i.e. $\left|t_{i, j}\right|=\delta_{i, j}$, with different phase factors:

$$
\hat{\Phi}=\left(\begin{array}{cc}
\mathrm{e}^{\mathrm{i} \phi_{0}} & 0 \\
0 & \mathrm{e}^{\mathrm{i} \phi_{1}}
\end{array}\right),
$$

corresponding to $\mathbf{R}_{\mathbf{z}}$, up to a global phase factor. In the solidstate implementations based on ESs, this operation is generally achieved by forcing the two edge channels to follow different tunable paths $\left(l_{0}, l_{1}\right)$ in the 2DEG. Electrons accumulate a phase $\phi_{i}=\frac{1}{\hbar} \int_{i}(p-q A) \mathrm{d} s_{i}=\xi_{i}+\chi_{i}$, that depends on the line integral of the total momentum on the specific path of the edge channel, $i=0,1$. This phase $\phi_{i}$ is split into a dynamical $\left(\xi_{i}\right)$, and a magnetic $\left(\chi_{i}\right)$ contribution depending on the linear and magnetic momentum, respectively. The dynamic phase difference between the paths of the two channels $i=0,1$ reads:

$$
\Delta \xi=\xi_{1}-\xi_{0}=\frac{1}{\hbar}\left(\int_{l_{1}} p \cdot \mathrm{d} r-\int_{l_{0}} p \cdot \mathrm{d} r\right)=-k \Delta l,
$$

with $\Delta l=l_{1}-l_{0}$ length difference between the two paths, and the relative magnetic phase

$$
\Delta \chi=\chi_{1}-\chi_{0}=\frac{e}{\hbar} \int_{S} \nabla \times A \cdot \mathrm{d} s=\frac{e}{\hbar} B S=\pi \frac{\Phi_{B}}{\Phi_{0}}
$$

is computed from the flux of the magnetic field through the area enclosed by the two channels $(S)$ via the Stoke's theorem; $\Phi_{0}=\frac{h}{2 e}=2.0678 \times 10^{-15} \mathrm{~Wb}$ is the magnetic flux quantum.

Then, in order to define a loop area $S$ and a path difference $\Delta l$ at bulk FF 1 , the geometry of an Hall phase shifter must force the two channels to propagate in the same direction. This is obtained, for example, with the potential landscape mapped in figure 5(a). Here, the phase shifter is integrated into a full MZI. Two QPCs link a potential mesa with local FF 0 (as in the confining potential) to an indentation with local FF 1 (as in the bulk) inside the confining barrier of the 2DEG. The initial state (red WP of figure 5(a)) is split by the first QPC into a reflected (blue arrow) and transmitted (red arrow) component that are counterpropagating on the lateral barriers of the mesa (for negative $k$ ) and the indentation (for positive $k$ ), due to the opposite slope of the potential in this horizontal path. In absence of additional energy-degenerate states, the two channels follow the border of each confining potential without additional interchannel scattering. ESs with $\Delta k<0$ are therefore confined at the outer ${ }^{3}$ edge of the indentation, while the state with $\Delta k>0$ originate at the inner edge of the potential mesa. At the end of the potential mesa, where the inner channel and outer channel are recollected at the second QPC, the reflected component of the final state propagates back inside the indentation zone, so that it can not be reused as an input for a possible following stage of quantum gates. This topological limit prevents the scalability of Hall interferometers at bulk FF 1 and its concatenation for many-qubit architectures. For this reason, the back-reflected channel is generally absorbed by an Ohmic contact at the inner edge of the indentation/ outer edge of the potential mesa (orange shape in figure 5(a)), which poses a limit to the miniaturization of the loop area.

On the contrary, at bulk FF 2 the output basis states/ channels stem at the same side of the device, where they are characterized by a different energy dispersion $E_{n}(k)$, with $n=0,1$. At a given energy $E$, the different wave vectors $k^{n}$ involve the localization of the two ESs $\varphi_{n, k}(x)$ around separated centers $x_{0}^{n}$. Their spatial separation is further increased by applying top gates to locally vary the depletion of the 2DEG, or equivalently the FF. Figure 5(b) shows the potential profile of the phase shifter in this scenario. The dark gray

3 In our convention, inner and outer refer to the center of the confined 2DEG. 
(a)

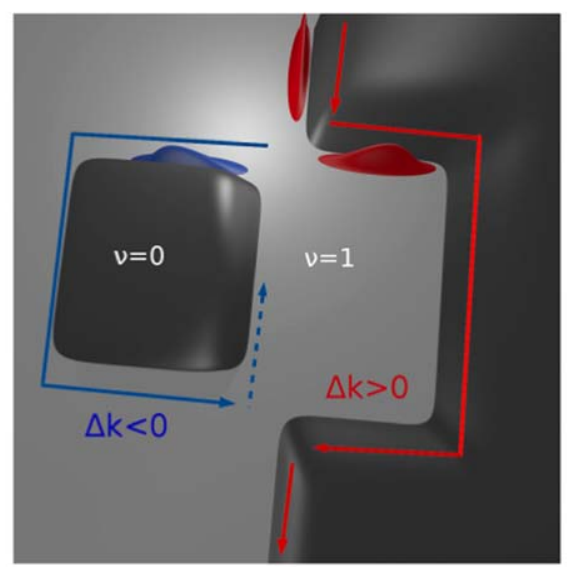

(b)

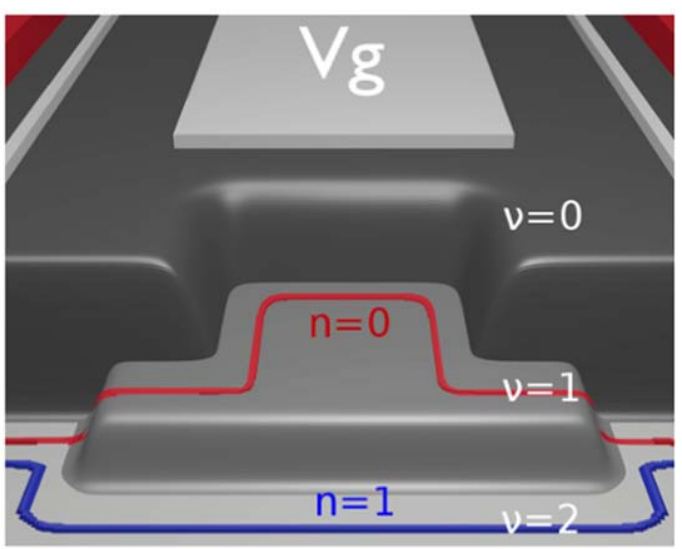

Figure 5. Rendered picture of possible geometries for the phase shifter. (a) Potential profile of the MZI based on counterpropagating ESs, at bulk FF 1 . The phase shifter is realized by the square potential mesa $(\nu=0)$ and indentation $(\nu=1)$ between the two QPCs. The red arrow (outer edge of the hole) indicates the electron path in channel 0, while the blue arrow (inner edge of the step) the electron path in channel 1. (b) Potential profile of the confining barrier for the phase shifter based on copropagating ESs, at bulk FF 2. The additional mesa with bulk filling factor 1 separates the first edge channel (red line, running along the inner edge) to the second one (blue line, running along the outer edge).

region is the lateral confining barrier with zero $\mathrm{FF}$, while the light gray region is an additional potential step with FF 1 . The two channels are forced to propagate on a different edge of the potential mesa, according to their Landau index: the inner channel $(n=1)$ propagates at the bottom of the inner edge, while the outer channel $(n=0)$ on top of the mesa at the outer edge. The width of the potential step controls the relative path of the two channel, $\Delta l$, together with the area enclosed by them. This geometry solves the scalability issue of the previous phase shifter, but it requires a careful engineering of the potential mesa in order to avoid additional interchannel mixing. The present geometry also entails different group velocities for electrons propagating in the two channels, and care must be taken to include this effect in the modeling of the system [69]. For example, in the potential landscape reported in figure 5(b), an indentation in the lateral confining barrier extends the path of the outer ES to compensate its higher velocity with respect to the inner one.

In realistic implementations of Hall interferometers, where non-monochromatic beams of electrons or energybroadened WPs are injected, the energy dependence of phase shifting must be accounted for in both scenarios. Indeed, the line and surface integrals in equations (31) and (32) depend on the distribution of the ES in the transverse direction, which is related to the energy-dependent center $x_{0}^{n}(E)$. The interference pattern is then affected by phase averaging on large energy windows [96]. This is a detrimental effect on the visibility of the device, that may arise also in absence of decoherence induced by impurities or thermal smearing. Giovannetti et al [97] propose a protocol to discriminate between the two sources of reduction in visibility by connecting two multi-channel MZIs - the only scalable geometry available. The decrease in visibility is enhanced in singlechannel geometries with respect to multi-channel ones, due to the larger size entailed by the inner Ohmic contact [7, 8, 51, 98]. The larger loop area, about 2 orders of magnitude, increases indeed the effect of area and flux fluctuations, thus making multi-channel geometries of the MZI even more suitable for quantum computing.

\section{3. $B S$ at FF 1: $Q P C$}

The realization of a coherent superposition between the two basis states requires the BS. This transforms the initial state of the qubit $i=0,1$ into a combination of the two basis states $j=0,1$ with a (generally complex) transmission coefficient $t_{i j}$. The transformation is represented by the following matrix:

$$
B=\left(\begin{array}{cc}
t_{00} & t_{01} \\
t_{10} & t_{11}
\end{array}\right)
$$

where $\left|t_{i j}\right|=1 / \sqrt{2}$ for ideal interferometers.

The earliest BSs in Hall devices have been realized with a QPC, a narrow constriction in the 2DEG that back-reflects the impinging electrons. In the IQH regime at bulk FF 1, an ES $\varphi_{n, k}$ can be scattered only to the counterpropagating one with opposite chirality, $\varphi_{n, k}$. This state is located in general at the opposite side of the device, so that the confining barriers must be brought near to each others to favor the interchannel scattering. Then, when the two borders are further separated, the wave function splits between the two paths with a transmission probability $\left|t_{i j}(E)\right|^{2}$ from the input $(i=0,1)$ to the output $(j=0,1)$ channel that depends on the energy of the state.

Due to the topological limit affecting Hall interferometers with counterpropagating channels, this type of BS has been initially integrated mainly into Fabry-Perot interferometers [4, 5, 99-101], while its experimental realization in semiconductor MZIs is more recent [8, 51, 102]. An efficient engineering of the MZI requires indeed a suitably small loop area to observe the interference pattern with a relevant visibility, which can be strongly quenched by decoherence effects related to the geometry of the phase shifter.

The energy selectivity of the QPC has a significant role on device performances if strongly localized WPs are used. 
Numerical simulations of electron scattering at a full-scale QPC in Hall semiconductors [67, 103] provide an empirical formula for the transmission $t(k)$ and reflection $r(k)$ coefficients of the BS:

$$
\left[\begin{array}{c}
r\left(k^{\prime}\right) \\
t\left(k^{\prime}\right)
\end{array}\right]=\sqrt{\frac{1}{\exp \left( \pm \alpha\left(k^{\prime}-k_{e}\right)+1\right)}},
$$

where $k$ is the wave vector of the impinging electron, $k_{e}$ and $\alpha$ are fitting parameter dependent on the geometry of the device, e.g. the distance between the two dips and the smoothness of the lateral barrier. Due to its Fermi-like selectivity, the QPC transmits mainly high-energy scattering states and reflects the low-energy ones, with respect to its central wave vector $k_{e}$. In the case of a localized WP of ESs with $k_{0}=k_{e}$, this translates into the redistribution of high and low energy components into two localized WP peaked around different centers. Therefore, the transmitted and reflected portion of the wave function are not identical in the wave vector space and overlap only partially.

For Gaussian WPs of ESs, the coefficients in equation (4.3) are well described by a Gaussian distribution:

$$
\sqrt{\frac{1}{\exp \left( \pm \alpha\left(k^{\prime}-k_{e}\right)+1\right)}} \simeq \exp \left[-\frac{\left(\alpha\left(k^{\prime}-k_{e}\right)+\gamma\right)^{2}}{4 \gamma}\right]
$$

with $\gamma=4 \ln (2)$. Within this approximation, the very high or very low energies are over- or under-estimated, respectively. However, for a large electron WP centered around $k_{e}$, such contributions are in the tails of the single-electron WP, so that they does not affect significantly the scattering process at the QPC. This model has indeed been applied to predict the visibility of a single-channel MZI [103], and the bunching probability in two-particle HOM experiments [88] with strongly localized WPs.

\subsection{BS at FF 2: potential dip or magnetic fingers}

At bulk FF larger than 1, the first two energy-degenerate ESs can be used to encode the qubit state. Namely, the state with Landau index $n=0(n=1)$ represents the qubit state $|0\rangle$ $(|1\rangle)$. In this case, elastic interchannel mixing implements the quantum logic gate $\mathbf{R}_{\mathbf{x}}$ of equation (2). In this section, we will consider a device operating at bulk FF 2, and the presence of carriers in higher LLs will be considered, from the quantum information processing perspective, a leakage from the computational space. The scattering between copropagating ESs requires, for example, a potential sharpness on their path $[104,105]$, that extend on a scale of a few magnetic lengths [106]. For $B=5 \mathrm{~T}$, this length is about $10 \mathrm{~nm}$, which is certainly feasible with nowadays technologies. Thus, interchannel scattering induced by a short-scale potential variation becomes a valid alternative to the mixing of counterpropagating states induced by QPCs illustrated in the previous section. The former solves indeed the topological limit affecting nanointerferometers at bulk FF 1.

Earliest experiments of electron BSs at bulk FF 2 used the design of abrupt potentials-called nanofingers - on the path of the ES to generate inter-channel scattering. However, without a fine shaping of its geometry, the potential dip acts as a BS with a low transmission coefficient, far from the target of 50\% [106]. Multiple reflection by arrays of nanofingers were initially exploited in quasi-Corbino [107, 108] and quasi-Fabry-Perot interferometers [100], to increase the overall transmission probability. These devices are still affected by a topological limit in scalability, as for counterpropagating ESs: the inner edge channel $(n=1)$ is confined inside the loop area, where it must be absorbed by an Ohmic contact. Later, numerical simulations showed that a significant enhancement in the transmission probability can be achieved when the spatial periodicity among the nanofingers matches the resonant condition [106]. This has been proven experimentally by using spin-resolved ESs, where an additional periodic in-plane magnetic field flips the edge spin to generate inter-channel scattering [52]. However, due to the large dimensionality of the array, the two channels copropagate for long distances next to each other, thus favoring decoherence induced by charge fractionalization [55, 98, 109].

Differently, in [69] the full-scale geometry of a single nanofinger is numerically designed to act as an half-reflecting BS for a single-electron WP injected in the MZI. Indeed, an array of nanofingers would not be a viable technology for cyclotron-resolved ESs, due to the smaller spatial periodicity required (few $\AA$ ) by the resonant condition [110]. The fine tuning of the sharpness must take into account the energy dispersion of the scattered state. For a WP of ESs, an ideal $\mathrm{BS}$, in addition to induce an integrated transmission probability of $1 / 2$, should produce identical reflected and transmitted WPs in the $k$ space. This requirement is necessary to usefully integrate the logic gate in a MZI. Indeed, all the transmitted and reflected components of the initial WP must overlap in $k$ space, in order to cancel each other completely after the interference at the second BS. Reference [69] reports a specific steep geometry for the nanofinger that generates a quasi-flat energy selectivity on the energy broadening of the initial WP. Its numerical simulation in a multi-channel MZI shows indeed a significantly higher visibility of $\mathrm{AB}$ oscillations with respect to alternative scenarios at bulk FF 1, where the energy selectivity of the QPC significantly decreases device performances [103].

\subsection{Creating entanglement: conditional phase shifter}

Together with the electron BS and phase shifter for singlequbit logic operations, a complete set of quantum logic gates requires the engineering of a two-qubit device able to generate maximal entanglement from a product state. This coupling is realized by the conditional phase shifter $\mathbf{T}$, of equation (6), which selectively adds a phase $\gamma$ to a specific two-qubit basis state.

In a solid-state implementation of the conditional phase shifter, Coulomb interaction acts as the coupling effect in the $\mathbf{T}$ transformation. In fact, when two electrons in channel $i$ and channel $j$ approach each other in presence of electron-electron repulsion, part of their kinetic energy is transformed into 


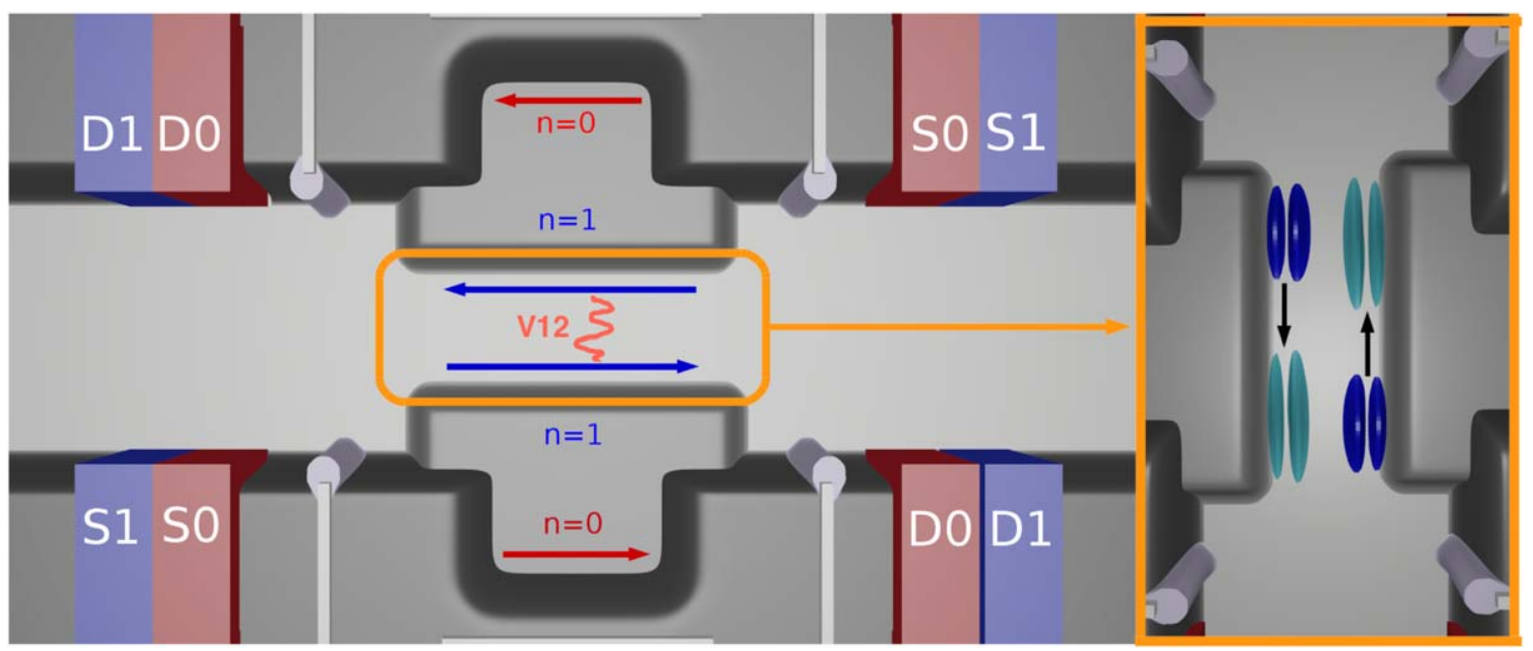

Figure 6. Two parallel MZIs define a conditional phase shifter $\mathbf{T}$ in the IQH regime. The two channels with Landau index $n=1$ interact in the active region (squared box), while the channels with $n=0$ are separated by increasing the width of the region at FF 1 . The inset shows the time evolution of the density probability of each particle in the $n=1$ channels.

a repulsive coupling potential and the velocity along their path is reduced. Then, when their relative distance returns to the original value, the potential energy is transformed back into kinetic energy and the initial velocity is restored. As a result of this process, the two electrons will undergo a delay in their propagation compared to the non-interacting case. This delay corresponds to a phase factor in front of the $|i j\rangle$ component of the two-qubit wave function, and it depends on the geometrical parameters of the system, e.g. the length of the coupling region and the distance between the two electrons.

At bulk FF 2, for the two counterpropagating electrons four edge channels are energetically available in the 2DEG, two for each side of the device with Landau indexes $n=0,1$. The system can be engineered as two MZIs connected in parallel, with the mesa structures facing each others, as in our proposal for a solid-state implementation of the device. Figure 6 shows a viable geometry based on the multichannel MZI simulated in [69]. Here, by tuning the width of the loop area, the two external channels $(n=1)$ are brought near to each other, and their mutual interaction is increased. On the contrary, the channels closer to the potential edge barrier $(n=0)$ can be separated by locally depleting the confining barrier, so that their interaction with the states of the other electron is negligible. The inset of figure 6 shows the initial (blu shapes) and final (light blu shapes) probability distribution of the two interacting WPs in the coupling region, which is targeted by the yellow square in the full-scale device, resulting from our time-dependent modelling of the twoelectron dynamics.

Numerical simulations of the proposed scheme prove that long-range Coulomb interaction acts as a selective entangler in this geometry (figure 7). Two Gaussian WPs are initialized at opposite sides of the active region (blue WPs in the inset of figure 6) in the second LL. Figure 7(a) shows the transverse probability distribution of the right WP at initial time. Here, the overlap between the two WPs, symmetrically distributed along the $\hat{x}$-direction, is negligible, thus ensuring the absence of elastic tunneling between the two channels. The spatial shift $\delta l$ induced by the electron interaction is obtained by comparing the final position of the two WPs in presence of Coulomb repulsion with the non-interacting case. Such shift can be increased/decreased by moving closer/farther the edges of the two potential mesas, or by tuning the smoothness of the LL. $V(x)$ is indeed engineered in order to induce a quasi-parabolic dispersion of the second LL (orange dashed curve in figure 7(a)), rather than a quasi-linear one, so that a larger $\delta l$ is expected. In case of a linear dispersion, indeed, the kinetic energy of the two WPs are initially decreased and finally increased by the same amount, so that the effect of electron-electron repulsion would not be detectable.

The phase shift $\gamma$ in the $\mathbf{T}$ matrix is related to the spatial shift $\delta l$ by resorting to the longitudinal separation $L_{\text {eff }}$, which is necessary to produce a $2 \pi$ rotation in a single-electron MZI. Figure 7(b) reports the transmission probabilities of Gaussian WPs initialized in the output state of the quasi half-reflecting BS in section 4.3 (a coherent superposition of WPs in channel 0 and 1$)$, scattered on a second identical electron BS, and then measured trough its probability of being detected in the output channel 0 or 1 . The scattering events have been dynamically simulated with the single-particle version of our software, for Gaussian WPs with different spatial broadenings $\sigma$; the two components of the single-electron initial wave function are delayed by a spatial factor $\Delta y$, in order to reproduce the interference pattern in a full-scale MZI (section 5.2).

The periodicity $L_{\text {eff }}$ of the interference fringes in figure 7 (b) corresponds to a $2 \pi$ qubit rotation in the output state of the Mach-Zehnder experiment. The space shift induced by the Coulomb interaction, $\delta l$, for two interacting electrons in channel $n=1$ can therefore be related to an equivalent phase shift $\gamma$ by using $L_{\text {eff }}$ as a reference:

$$
\gamma=2 \pi \frac{\delta l}{L_{\mathrm{eff}}}
$$

For two WPs with $\sigma=40 \mathrm{~nm}$ and a separation between the two mesas $W=100 \mathrm{~nm}$ in the active region, our two-particle 

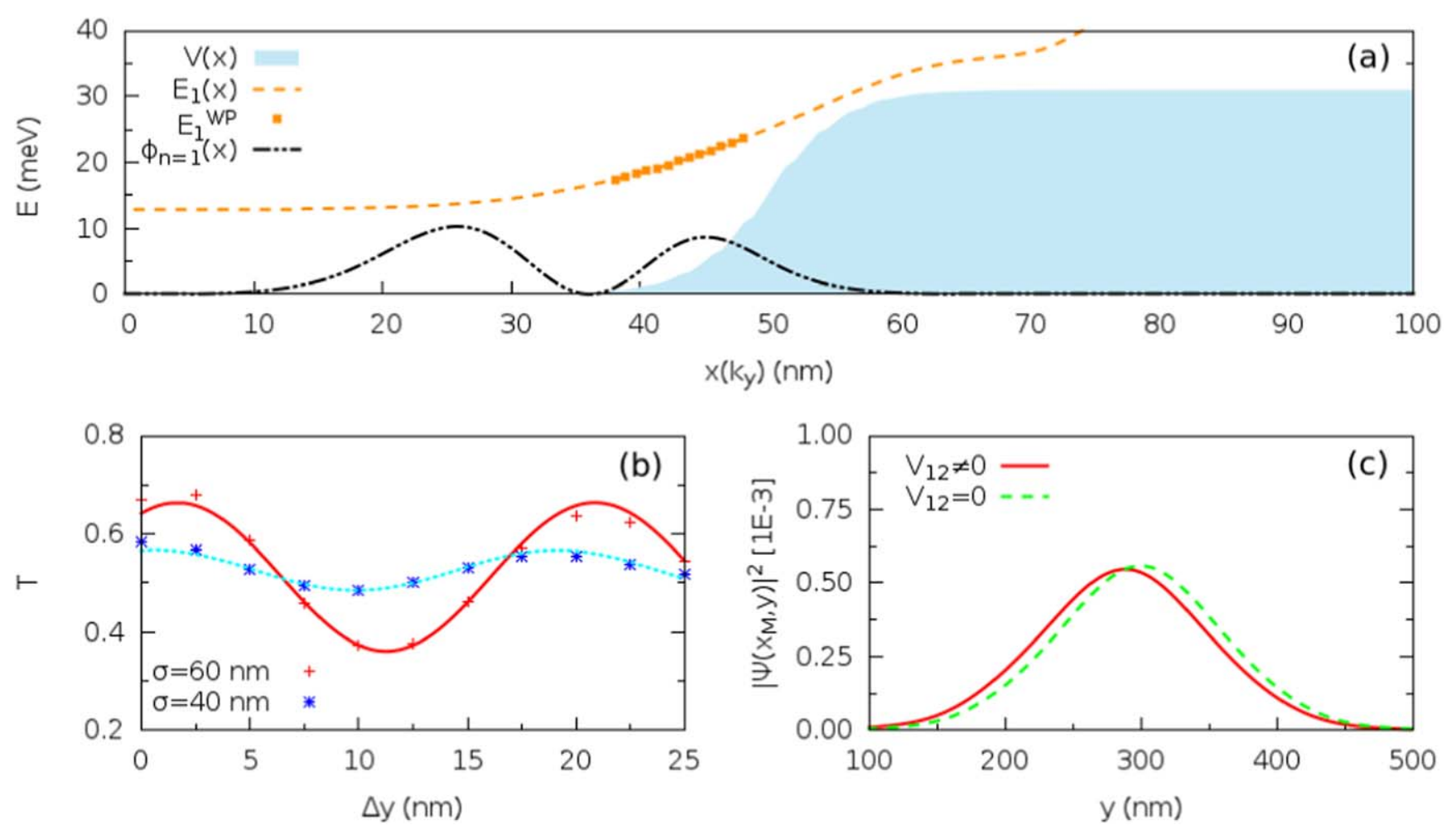

Figure 7. (a) Band structure of the system at the central coordinate $y_{0}$ for the right WP in the inset of figure 6 . The transverse probability distribution of the electron WP in the second $\left(\phi_{n=1}\right)$ edge channel is compared to the energy dispersion of the second $\left(E_{1}(x)\right)$ LL. (b) AB oscillations of the MZI for a geometrical configuration where only the longitudinal distance between the two WPs in the phase shifter is varied. T measures the probability that the electron state is transmitted to the output channel 0 , for different spatial broadenings of the WP $\sigma=60,40 \mathrm{~nm}$. (c) Comparison between the longitudinal probability distribution of one of the two WPs in the inset of figure 6 at final time with (red solid line) and without (green dashed line) electron-electron interaction for $x=x_{M}$, where $x_{M}$ is the maximum of the probability distribution in the $\hat{x}$-direction.

simulations in presence of long-range Coulomb repulsion produce a $\delta l=11 \mathrm{~nm}$ with respect to the non-interacting case, as shown in figure 7(c). According to equation (36), this is equivalent to a $\gamma=\pi$ rotation for the effective length $L_{\text {eff }}=19 \mathrm{~nm}$ of the interference pattern in figure $7(\mathrm{~b})$.

The relative distance between the two mesas required for a $\pi$ rotation in our numerical simulations, i.e. $W=100 \mathrm{~nm}$, is feasible with current nanotechnology. This validate the present geometry as a phase shifter in the IQH regime, where the phase can be efficiently tuned up to e.g. $\pi / 4$ by simply increasing the distance between the two mesas on such achievable length scales.

\section{Dynamics of a single carrier: time-dependent numerical simulation}

\subsection{Propagation method}

In the present section we describe the numerical method adopted for the time propagation of the one- and two-particle state in presence of a magnetic field [67, 111-114]. For a system described by the single-particle Hamiltonian $\hat{H}=\hat{T}+\hat{V}$, the evolution of the wave function is computed from the time-dependent Schrödinger equation

$$
\mathrm{i} \hbar \frac{\partial \Psi}{\partial t}=\hat{H} \Psi
$$

Equation (37) can be solved by applying to the initial wave function $\Psi(x, y ; t=0)$ the evolution operator

$$
\hat{U}(\delta t)=\mathrm{e}^{-\frac{\mathrm{i}}{\hbar} \delta \cdot \hat{H}} .
$$

For a time interval short enough, $\delta t \rightarrow 0$, one can apply the Trotter Suzuky factorization to separate the kinetic and potential operator:

$$
\begin{gathered}
\hat{U}(\delta t)=\mathrm{e}^{-\frac{\mathrm{i}}{\hbar} \delta t \cdot(\hat{T}+\hat{V})} \\
=\mathrm{e}^{-\frac{\mathrm{i}}{\hbar} \delta t \cdot \frac{\hat{V}}{2}} \mathrm{e}^{-\frac{\mathrm{i}}{\hbar} \delta t \cdot \hat{T}} \mathrm{e}^{-\frac{\mathrm{i}}{\hbar} \delta t \cdot \frac{\hat{V}}{2}} \mathrm{e}^{O\left(\delta t^{3}\right)} .
\end{gathered}
$$

The last term, representing a small numerical error depending on $\delta t^{3}$, can be ignored. It is worth remembering that the systems considered in the present work are $2 \mathrm{D}$, so that only the coordinates $x$ and $y$ in the real space, and $k_{x}, k_{y}$ in the reciprocal space, need to be considered. The potential operator $\hat{V}$ is diagonal in the real space, while the kinetic operator

$$
\hat{T}=\hat{T}_{p_{x}}+\hat{T}_{p_{y}}=\frac{\hbar^{2}}{2 m^{*}} k_{x}^{2}+\frac{\hbar^{2}}{2 m^{*}} k_{y}^{2}
$$

is diagonal in the wave vector space. $m^{*}$ is the effective carrier mass and, for simplicity, will be considered the same 
for the two directions. It is therefore convenient to apply the exponential of the kinetic operator by using its representation in the reciprocal space, where $\hat{T}$ is a scalar function. To this purpose, the kinetic evolution operator must be applied to the wave function in the wave vector space representation, which is obtained from $\Psi(x, y)$ by a Fourier transform:

$\mathcal{F} \Psi(x, y)=\frac{1}{(2 \pi \hbar)} \int_{-\infty}^{+\infty} \mathrm{e}^{-\frac{\mathrm{i}}{\hbar} p \cdot r} \Psi(x, y) \mathrm{d} r=\tilde{\Psi}\left(k_{x}, k_{y}\right)$.

The evolution operator will be therefore split in two parts, potential and kinetic, applied to the wave function in the real space and reciprocal representation, respectively. Including the forward and backward Fourier transforms, the evolution operator reads

$$
\hat{U}(\delta t)=\mathrm{e}^{-\frac{\mathrm{i}}{\hbar} \delta t \cdot \frac{\hat{V}}{2}} \mathcal{F}^{-1} \mathrm{e}^{-\frac{\mathrm{i}}{\hbar} \delta t \cdot \hat{T}} \mathcal{F} \mathrm{e}^{-\frac{\mathrm{i}}{\hbar} \delta t \cdot \frac{\hat{V}}{2}} .
$$

In order to evolve the wave function for a time $t=N \delta t$, the operator of equation (43) must be applied for $N$ time steps, so the evolution of the wave function will be given numerically by:

$$
\Psi(x, y ; t)=[U(\delta t)]^{N} \Psi(x, y ; 0) .
$$

To lower the computational cost, the $N$ time steps can be rearranged in the following way:

$$
\Psi(x, y ; t)=\mathrm{e}^{\frac{\mathrm{i}}{\hbar} \delta t \cdot \frac{\hat{v}}{2}}\left(\mathrm{e}^{-\frac{\mathrm{i}}{\hbar} \delta t \cdot \hat{V}} \mathcal{F}^{-1} \mathrm{e}^{-\frac{\mathrm{i}}{\hbar} \delta t \cdot \hat{T}} \mathcal{F}\right)^{N} \mathrm{e}^{-\frac{\mathrm{i}}{\hbar} \delta t \cdot \hat{\hat{V}}} .
$$

In presence of a magnetic field, the momentum $\mathbf{p}$ is replaced by $\mathbf{p}-q \mathbf{A}$, where $\mathbf{A}$ is the magnetic vector potential obtained from $\mathbf{B}=\nabla \times \mathbf{A}$. We will consider a magnetic field applied along the $\hat{z}$ direction, i.e. orthogonal to the $2 \mathrm{DEG}$ plane. In the Landau gauge, $\mathbf{A}=(0, x B, 0)$ so that

$$
\begin{aligned}
\hat{T}= & \frac{1}{2 m^{*}} \sum_{i=x, y, z}\left(p_{i}-q A_{i}\right)^{2}=\frac{\hbar^{2}}{2 m^{*}}\left(k_{y}-\frac{e B x}{\hbar}\right)^{2} \\
& +\frac{\hbar^{2}}{2 m^{*}} k_{x}^{2}=\hat{T}_{p_{y}, x}+\hat{T}_{p_{x}, y} .
\end{aligned}
$$

Here, the Fourier transform acts on a single coordinate at a time. The time-evolved wave function is

$$
\begin{aligned}
\Psi(x, y ; t)= & \mathrm{e}^{\frac{\mathrm{i}}{\hbar} \delta t \cdot \frac{\hat{V}}{2}}\left(\mathrm{e}^{-\frac{\mathrm{i}}{\hbar} \delta t \cdot \hat{V}} \mathcal{F}_{y}^{-1} \mathrm{e}^{-\frac{\mathrm{i}}{\hbar} \delta t \cdot \hat{T}_{p_{y}, x}} \mathcal{F}_{y} \mathcal{F}_{x}^{-1} \mathrm{e}^{-\frac{\mathrm{i}}{\hbar} \delta t \hat{T}_{p_{x}}} \mathcal{F}_{x}\right)^{N} \\
& \times \mathrm{e}^{-\frac{\mathrm{i}}{\hbar} \delta t \cdot \frac{\hat{v}}{2}} \Psi(x, y ; 0) .
\end{aligned}
$$

The algorithm can be straightforwardly extended to the two-particle case as follows. Without the magnetic field, the two-particle Hamiltonian $H\left(x_{1}, y_{1}, x_{2}, y_{2}\right)=H_{1}\left(x_{1}, y_{1}\right)$ $+H_{2}\left(x_{2}, y_{2}\right)+V_{\text {int }}\left(x_{1}, y_{1}, x_{2}, y_{2}\right)$ can be rearranged as

$H\left(x_{1}, y_{1}, x_{2}, y_{2}\right)=T\left(x_{1}, y_{1}, x_{2}, y_{2}\right)+V\left(x_{1}, y_{1}, x_{2}, y_{2}\right)$,

where $\quad T\left(x_{1}, y_{1}, x_{2}, y_{2}\right)=T_{1}\left(x_{1}, y_{1}\right)+T_{2}\left(x_{2}, y_{2}\right)$ and $V\left(x_{1}, y_{1}, x_{2}, y_{2}\right)=V_{1}\left(x_{1}, y_{1}\right)+V_{2}\left(x_{2}, y_{2}\right)+V_{\text {int }}\left(x_{1}\right.$, $\left.y_{1}, x_{2}, y_{2}\right)$. Equation (43) is can be rewritten by using 4D Fourier transform on the $\left(x_{1}, y_{1}, x_{2}, y_{2}\right)$ space:
$\hat{U}(\delta t)=\mathrm{e}^{-\frac{\mathrm{i}}{\hbar} \delta t \cdot \frac{\hat{V}\left(x_{1}, y_{1}, x_{2}, y_{2}\right)}{2}} \mathcal{F}_{4 D}^{-1} \mathrm{e}^{-\frac{\mathrm{i}}{\hbar} \delta t \cdot \hat{T}\left(x_{1}, y_{1}, x_{2}, y_{2}\right)} \mathcal{F}_{4 D} \mathrm{e}^{-\frac{\mathrm{i}}{\hbar} \delta t \cdot \frac{\hat{v}\left(x_{1}, y_{1}, x_{2}, y_{2}\right)}{2}}$.

The same holds for equation (47), where $\mathcal{F}_{x}\left(\mathcal{F}_{y}\right) \rightarrow \mathcal{F}_{x_{1}, x_{2}}\left(\mathcal{F}_{y_{1}, y_{2}}\right)$ and similarly for the antitransform. Indeed, with the presence of a magnetic field, the Landau gauge couples the linear momentum $\hat{p}_{y_{i}}$ and the position $\hat{x}_{i}$ of the same particle $i=1,2$, so that it is possible to perform 2D Fourier transforms on the $\left(x_{1}, x_{2}\right)$ and $\left(y_{1}, y_{2}\right)$ spaces. Equation (47) therefore reads:

$\Psi\left(x_{1}, y_{1}, x_{2}, y_{2} ; t\right)=\mathrm{e}^{\frac{\mathrm{i}}{\hbar} \delta t \cdot \frac{\hat{V}\left(x_{1}, y_{1}, x_{2}, y_{2}\right)}{2}}\left(\mathrm{e}^{-\frac{\mathrm{i}}{\hbar} \delta t \cdot \hat{V}\left(x_{1}, y_{1}, x_{2}, y_{2}\right)}\right.$

$\left.\mathcal{F}_{y_{1}, y_{2}}^{-1} \mathrm{e}^{-\frac{\mathrm{i}}{\hbar} \delta t \cdot\left(\hat{T}_{p_{1}, x_{1}}+\hat{T}_{p_{y_{2}}, x_{2}}\right)} \mathcal{F}_{y_{1}, y_{2}} \mathcal{F}_{x_{1}, x_{2}}^{-1} \mathrm{e}^{-\frac{\mathrm{i}}{\hbar} \delta t \cdot\left(\hat{T}_{p_{x_{1}}}+\hat{T}_{p_{x_{2}}}\right)} \mathcal{F}_{x_{1}, x_{2}}\right)^{N}$

$\mathrm{e}^{-\frac{\mathrm{i}}{\hbar} \delta t \cdot \frac{V\left(x_{1}, \hat{y_{1}}, x_{2}, y_{2}\right)}{2}} \Psi(x, y ; 0)$.

The Split-Step Fourier method requires the discretization of both direct and reciprocal spaces. On a 2D simulation grid with $N_{x}$ and $N_{y}$ points distributed on a length $L_{x}, L_{y}$, the lattice parameters $\delta x=L_{x} / N_{x}$ and $\delta y=L_{y} / N_{y}$ are related to the discretization parameters on the $k$-space $\delta k_{x}=2 \pi / L_{x}$ and $\delta k_{y}=2 \pi / L_{y}$ by the following relations:

$$
\begin{aligned}
& k_{x}^{\max }=-k_{x}^{\min }=\frac{\pi}{\delta x} \\
& k_{y}^{\max }=-k_{y}^{\min }=\frac{\pi}{\delta y},
\end{aligned}
$$

so that also the reciprocal space is discretized in $N_{x} \times N_{y}$ points. Furthermore, the use of the Landau gauge imposes that $k_{y}=-\frac{e B x}{\hbar}$, and consequently for a system with the origin at the center of the device the maximum magnetic wave vector $\bar{k}_{y}^{\max }=\frac{e B L_{x}}{2 \hbar}$ must fulfill $\bar{k}_{y}^{\max }<k_{y}^{\max }$ in order to be correctly represented. Additionally, to correctly reproduce the WP dynamics, some care must be taken in the definition of the discrete time interval $\delta t$. In addition to be small with respect to typical dynamical times of the WP evolution, it must be smaller than the transition time between two grid points, which is defined by $\delta x / v_{g}$. Also, in order to avoid aliasing, the largest wave vector $k_{y}$ in the WP must be smaller than the maximum wave vector simulated by the grid in the $k$ space. Finally, $\delta t \ll \frac{2 \pi \hbar}{\max V}$.

\subsection{Mach-Zehnder interferometer}

In an electronic MZI the most basic building blocks for quantum computing, i.e. the BS $\mathbf{R}_{\mathbf{x}}$ and the phase shifter $\mathbf{R}_{\mathbf{z}}$, are combined to observe self-interference of the carrier wave function. The single-particle wave function is initially split into a coherent superposition of a reflected and a transmitted component by an half-reflecting BS. The phase shifter separates the two portions of the scattered state to introduce a relative phase, which can be tuned by the geometry of the system or the intensity of the magnetic field. Finally, the two beams are recollected on a second BS, where they interfere. In a solid-state implementation of the device, the visibility of $A B$ oscillations in the transmission amplitude measures the degree of coherence for single-electron transport, jeopardized by 
(a)

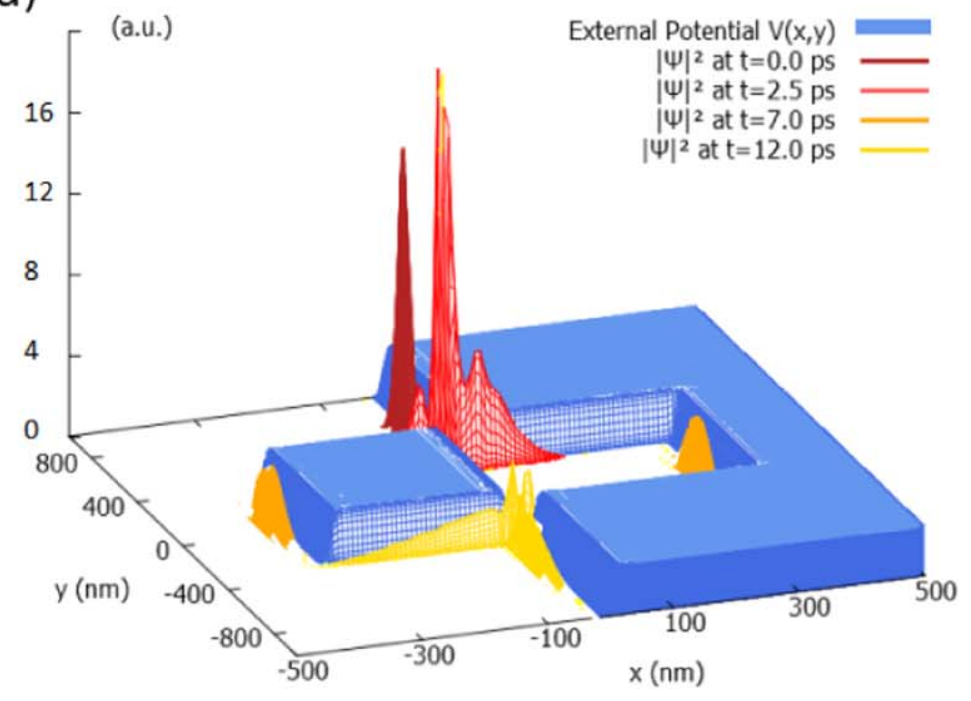

(b)

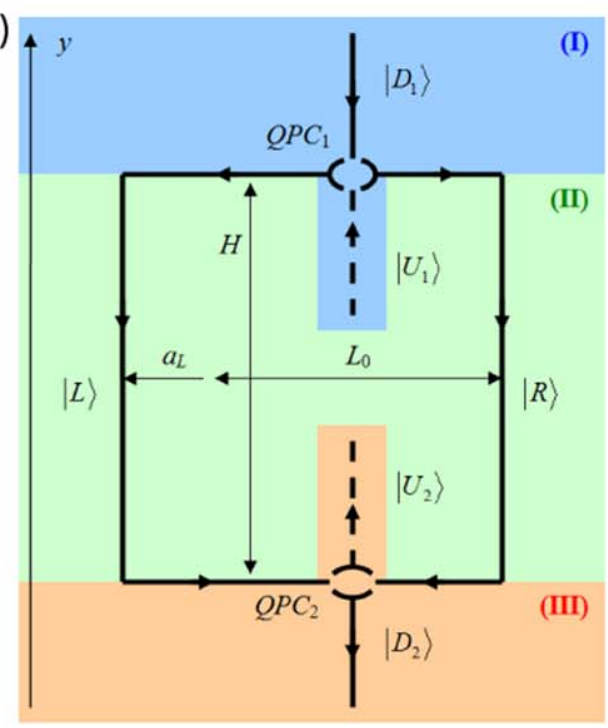

Figure 8. (a) Full-scale potential generated by top gates in the single-channel MZI of [103] (blue region) and dynamics of the single-electron WP. (b) Scheme and geometrical parameters of the MZI to implement the 1D effective model. The device is divided into 3 sections: (I) in blue, where the channel $\left|D_{1}\right\rangle$ is filled, (II) in green, where two paths $|L\rangle,|R\rangle$ propagate after the first QPC, and (III) in orange, where the final channel $\left|D_{2}\right\rangle$ — the only one transmitted outside region II by the second QPC—is measured.

typical decoherence processes induced by impurities $[115,116]$, phonons [117] or background charges.

Thanks to their long-range coherence length, ESs in the IQH proved to be ideal candidates to observe interference patterns in this device. The first experimental implementations of Hall MZIs were based on energy-degenerate counterpropagating states at integer FF, where only the first edge channel is available at each side of the 2DEG. The electron BS is realized with the QPC described in section 4.3 and the phase shifter is obtained with the step/indentation geometry described in section 4.2 (see figure 8(a)).

In the literature, the visibility of an electronic MZI is usually predicted by means of the analogy between ESs in 2D heterostructures and plane waves in 1D effective geometries. References [69, 103] generalize this result for localized WPs of ESs, and compare the theoretical predictions to the results of full-scale numerical simulations in a time-dependent framework (section 5.1). In this effective 1D scheme, the initial state is a linear combination of plane waves $\left|D_{1}\right\rangle$ along the $\hat{y}$ direction, $\left\langle y \mid D_{1}\right\rangle=\mathrm{e}^{\mathrm{i} k y}$ :

$$
\left|\Psi_{\mathrm{I}}\right\rangle=\int \mathrm{d} k F(k)\left|D_{1}\right\rangle,
$$

where $F(k)=N \exp \left(-\sigma^{2}\left(k-k_{0}\right)^{2}\right)$ is a Gaussian distribution centered at $k_{0}$ with spatial dispersion $\sigma$. The device is divided into three regions (figure 8(b)), containing the QPCs and the phase shifter, whose scattering matrices are detailed in section 2. The output state reads $\left|\Phi_{\text {III }}\right\rangle=\hat{B} \hat{\Phi} \hat{B}\left|\Psi_{\mathrm{I}}\right\rangle$. The final transmission probability for a single $k$-component of the WP in the final region, $\left|D_{2}\right\rangle$, has the well-known form [67]:

$$
\left|t^{\prime}(k)\right|^{2}=\left|\left\langle D_{2} \mid \Psi_{\mathrm{III}}\right\rangle\right|^{2}=T_{0}-T_{1} \cos \left(\Delta \chi-k_{0} \Delta l\right),
$$

where $T_{0}=\left|r_{1} r_{2}\right|^{2}+\left|t_{1} t_{2}\right|^{2}$ and $T_{1}=2 r_{1} r_{2} t_{1} t_{2}, \Delta \chi$ is the magnetic phase and $\Delta l$ the relative length difference between the two channel paths. By assuming (i) energy-independent coefficients $r_{i}(k)=t_{j}(k)=\frac{1}{\sqrt{2}}$ of the half-reflecting BS and (ii) an energy-independent phase $\Delta \chi-k_{0} \Delta l$, the total transmission probability for a Gaussian WP is computed analytically as

$T=2 \pi \int \mathrm{d} k|F(k)|^{2}\left|t^{\prime}(k)\right|^{2}=T_{0}-T_{1} \mathrm{e}^{-\frac{\Delta l}{8 \sigma^{2}}} \cos \left(\Delta \chi-k_{0} \Delta l\right)$.

The relative phase depends on the geometrical parameters of the MZI in figure $8(\mathrm{~b})$ :

$$
\begin{gathered}
\Delta \phi-k_{e} \Delta l=\left[\frac{e}{\hbar} H\left(a_{L}+L_{0}\right)\right] B+\varphi_{0} \\
=\left[\frac{e}{\hbar} H B-2 k_{e}\right] a_{L}+\varphi_{0},
\end{gathered}
$$

so that

$$
\begin{aligned}
T\left(a_{L}, B\right)= & T_{0}-T_{1} e^{-\frac{\left(a_{L}-L_{1}\right)^{2}}{2 \sigma^{2}}} \\
& \times \cos \left(\frac{e}{\hbar} B H\left(a_{L}+L_{0}\right)-2 k_{e}\left(a_{L}-L_{1}\right)\right) \\
= & T_{0}-T_{1} \mathrm{e}^{-\frac{\left(a_{L}-L_{1}\right)^{2}}{2 \sigma^{2}}} \cos \left(k_{a} a_{L}+\varphi_{a}\right) \\
= & T_{0}-T_{2} \cos \left(k_{B} B+\phi_{B}\right) .
\end{aligned}
$$

The total transmission probability of Gaussian WPs oscillates with the magnetic flux across the loop area and with the relative path difference, as in equation (53) for plane waves. However, in presence of a localized wave function, a change in the lateral dimension of the loop area $\left(a_{L}\right)$ induces also an exponential decrease in the visibility, which reads

$$
\text { vis }=\frac{\max (T)-\langle T\rangle}{\langle T\rangle} .
$$




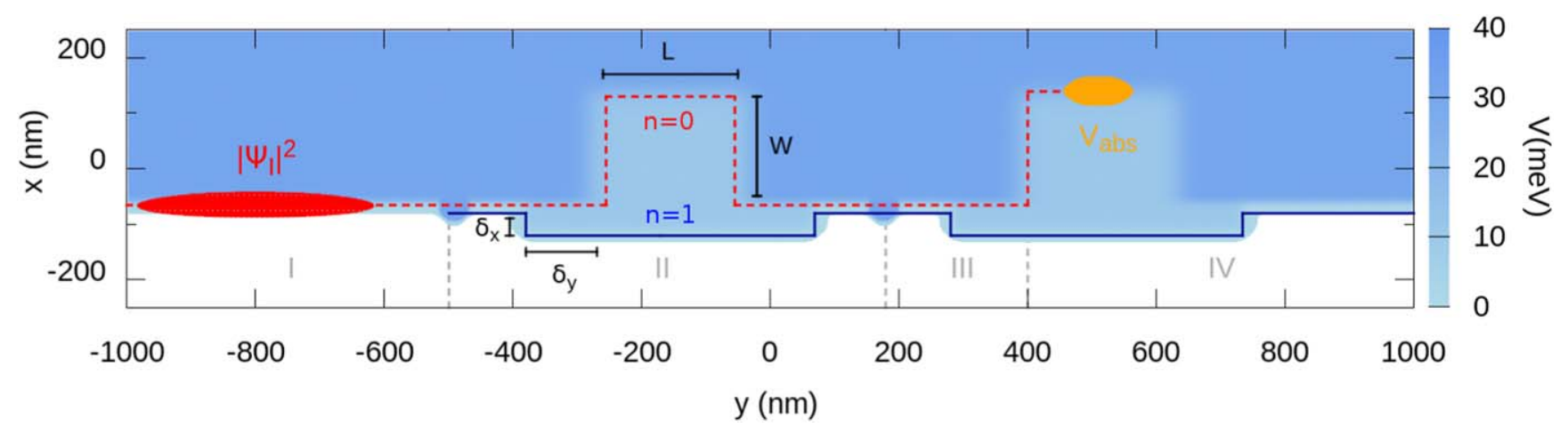

Figure 9. Top-view of the full-scale MZI as in [69]. the blue region is the external potential profile where the single-electron Gaussian WP (red shape) propagates.

The time-dependent approach described in section 5.1 provides a clear picture to explain a possible contribution to this visibility damping, which is related to charge localization $[62,103]$. The first BS splits the localized wave function in a reflected and transmitted components that are localized in space, too. Then, if the potential step and the indentation in the phase shifter have the same smoothness, the two components propagate with the same group velocity. For $\Delta l \neq 0$ they recollect at the second BS with a time delay induced by the path difference, and interfere accordingly to their relative phase. For a large path length difference, the two WPs do not overlap in space, so that the interference is suppressed. In contrast to the prediction of the simple theoretical model (equation (57)), numerical simulations in [103] show that the actual length scale for this process is larger than the spatial broadening of the WP $\sigma$. Moreover, this discrepancy is proved to decrease for $\sigma \rightarrow \infty$, that is when the electron WP is well approximated by a delocalized plane-wave with a single well-defined energy. This trend is typical of scattering processes where assumption (i) is adopted, as usually done in literature. The interplay between the energy broadening of the WP and the selectivity of the QPC can be accounted for by resorting to the empirical coefficients $r(k), t(k)$ (section 4.3); this leads to a definition of $T_{0}$ and $T_{1}$ that depends on the spatial broadening and geometrical parameters $\gamma$ and $\alpha$ :

$$
\begin{gathered}
T_{0}=\frac{\sigma}{2 \Sigma}, \\
T_{1}=2 \frac{\sigma}{\Sigma} \exp \left(-\frac{\gamma \sigma^{2}}{2 \Sigma^{2}}\right) .
\end{gathered}
$$

The visibility reads

$$
\text { vis }=\frac{1}{4} \exp \left(-\frac{\gamma \sigma^{2}}{2 \Sigma^{2}}\right)
$$

with $\Sigma^{2}=\sigma^{2}+\alpha^{2} / 4 \gamma$ the length scale for the damping of the transmission amplitude. The actual visibility is lower than the ideal value 1 , which is restored only in the limit $\alpha / 2 \sqrt{\gamma} \ll \sigma$. This condition describes indeed an electron as a delocalized plane wave, or a QPC with a selectivity smooth enough to neglect the energy dispersion of the WP.

However, assumption (ii) still holds in this model. The validity of such approximation depends on the energy broadening of the injected WP with respect to the smoothness of the energy dispersion $E_{n}(k)$ in the channel. For a large slope $a$, the states involved in the WP have a dispersion that is large in energy, but small in space, so that $x_{0}(k)$ is expected to be almost independent from $k$. When integrating over large energy windows, as typically done in steady-state scenarios, phase averaging represents a relevant source of decrease in visibility, as previously mentioned in section 4.2. To reduce the impact of assumption (ii), the injection of single-electron WPs with a high energy resolution is clearly a solution. However, phase averaging also depends on the miniaturization of the loop area, which is here limited by the presence of the Ohmic contact. Better performances are indeed expected in multi-channel implementations of the MZI, where the loop area can be decreased of 2 orders of magnitude with respect to the single-channel geometry.

At bulk FF 2, the basis consists of cyclotron-resolved ESs with a different energy dispersions $E_{n}(k)$ for each channel. Figure 9 displays the possible geometry of MZI investigated in [69]. The potential profile (blue region) mimics the effect of top gates in a realistic MZI. The shape of the BS (a single potential dip) is engineered in order to produce a smooth energy selectivity around 50\% (see section 4.4), while the phase shifter is a potential mesa with local FF 1 and smooth edges, so that additional interchannel mixing is avoided in this region. Here, the two components of the wave function propagate with a different group velocity $v_{g}^{n}$, that depends on the transverse potential profile of the mesa and on the Landau index $n$. The overlap between the two WPs at the second BS depends on the time difference between the propagation in the first and the second channel, from region I to region III: the highest overlap—and consequently visibilityis expected only when the two paths in region II, each divided by the corresponding group velocity, i.e. $\Delta s_{n} / v_{g}^{n}$, are the same for channel 0 and channel 1 . When a time delay between the two components of the wave function is present, an exponential decrease in the transmission amplitude from the first to the second channel (figure 10(b)) is observed.

This trend is confirmed analytically in [69] under the assumption of linear dispersions $E_{n}(k)$, which is a good approximation in the full-scale geometry under study. The 1D simplified model of equation (52) can be adapted to the present geometry, by assuming that an ES of the $n$th LL is represented by a plane wave along $y,|k, n\rangle$, with the (linear) 

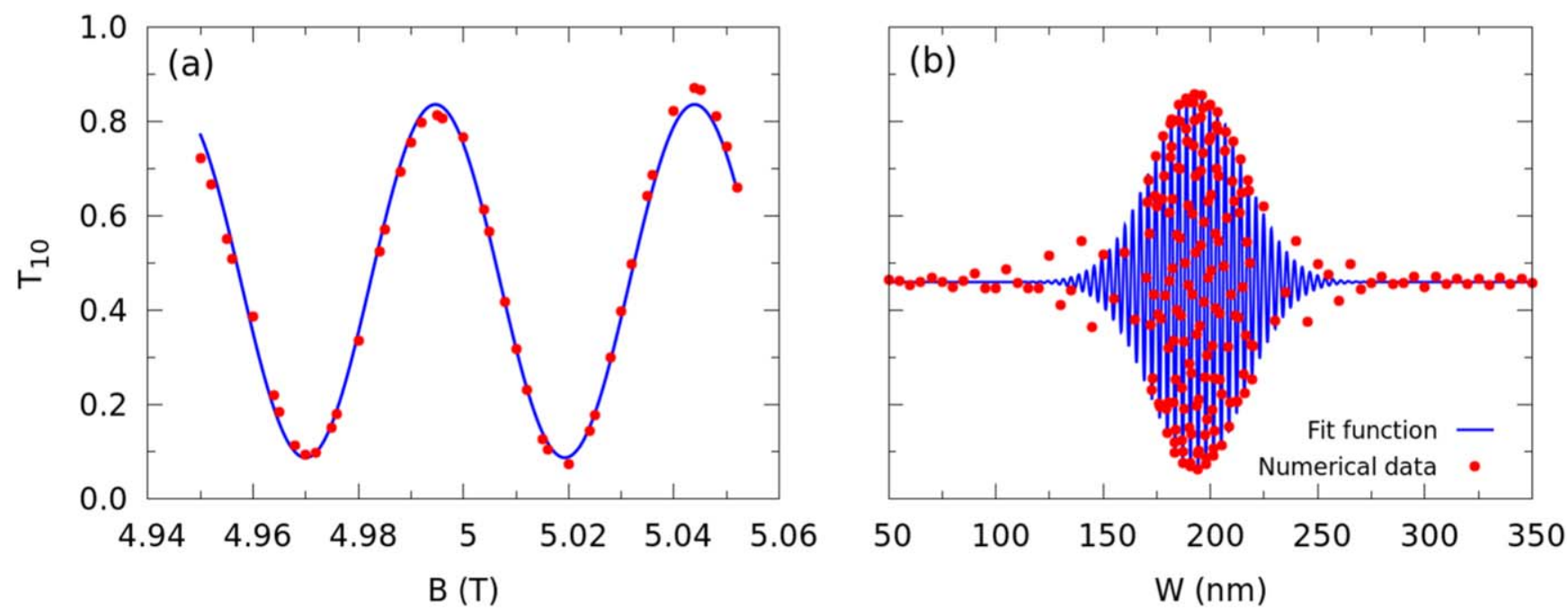

Figure 10. Transmission probability of a Gaussian WP initialized in channel $n=0$ and measured in channel $n=1$ with $\sigma=60 \mathrm{~nm}$. The amplitude oscillations are generated by a variation of the (a) magnetic field B and (b) width of the phase shifter $W$. Figures are adapted from [69].

energy dispersion of that $\mathrm{LL}, k(E, n)$. The dynamical phase is approximated as

$$
\chi_{i}=\int_{i} k \mathrm{~d} s=\frac{1}{\hbar} \int \frac{E-E_{0}}{v_{g}^{i}} \mathrm{~d} s=\frac{E-E_{0}}{v_{g}^{i} \hbar} \Delta s_{i},
$$

where $E_{0}$ is the central energy of the WP, $v_{g}^{i}$ the group velocity in region II and $\Delta s_{i}$, with $i=0,1$, is the path of channel $i$ in region II. Together with the magnetic contribution, the relative phase then reads:

$$
\Phi=\varphi+\frac{E-E_{0}}{\hbar}\left(\frac{\Delta s_{0}}{v_{g}^{0}}-\frac{\Delta s_{1}}{v_{g}^{1}}\right)+\frac{e B S}{\hbar},
$$

where $\Delta s_{0}, \Delta s_{1}$ and $S$ depend on the specific geometry of the device. At bulk FF 2, both channels reach the end of the device, where they can be used as input states for a following stage. In order to measure the transmission probability $T_{j i}$ from the input channel $i$ to one of the available output channels $j$, the two basis states can be selectively separated by smooth potential steps with a local FF 1 (region IV of figure 9 ), as in the phase shifter $[69,110]$. Figure 9 also sketches the measurement apparatus in the rightmost region labeled IV, where an additional potential mesa separates the two channels, and an imaginary potential absorbs the states in the first one. The component of the wave function with higher Landau index $(n=1)$ reaches the end of the device, where its probability amplitude corresponds to $T_{10}$ for an electron state initialized in the first LL. $T_{10}$ is computed from equation (54) in the multichannel geometry:

$$
\begin{aligned}
T_{10} & =\int\left|F(k(E)) \frac{\mathrm{d} k}{\mathrm{~d} E}\right|^{2}\left|t_{10}^{\prime}(E)\right|^{2} \mathrm{~d} E \\
& =\sqrt{2 \sigma^{2}} \pi \int \frac{\mathrm{e}^{-2 \frac{\sigma^{2}}{v_{g}^{0}}\left(E-E_{0}\right)^{2}}}{v_{g}^{0}}\left|t_{10}^{\prime}(E)\right|^{2},
\end{aligned}
$$

where the electron is initialized in a Gaussian WP of ESs with $n=0$ and $\overline{v_{g}}$ indicates the group velocity in region I. $t_{10}^{\prime}$ is the transmission coefficient for a single component of the WP, i.e. from equation (53),

$$
\left|t_{10}^{\prime}(E)\right|^{2}=2\left|t_{10}(E) t_{00}(E)\right|^{2}(1+\cos \Phi) .
$$

This expression holds under the hypothesis of ideal halfreflecting transmission coefficients $\left(\left|t_{00}(E)\right|^{2}=\left|t_{10}(E)\right|^{2}=1 / 2\right)$ and almost identical ESs $\varphi_{n k}(x) \sim \varphi_{n k_{0}}(x)$ (large $\sigma$ ). Note that, with a proper design of the electron BS-as in [69] - the first approximation is quite accurate, differently from the QPC in single-channel MZIs. The final transmission probability from the outer to the inner channel can be computed from equation (65):

$T_{10}=\frac{1}{2}\left[1+\exp \left(-\frac{\left(W-W_{0}\right)^{2}}{2 \Sigma^{2}}\right) \cos \left(\Phi^{\prime}\right)\right], \quad \Sigma=\frac{\sigma}{\bar{v}_{g}^{0} / \nu_{g}^{0}}$.

The analytical prediction of the simplified numerical model leading to equation (65) are confirmed by the full-scale numerical simulations of single-electron interference in the dynamical framework of [69]. Results are reported in figure 10. According to our 1D model, $T_{10}$ depends on an angle $\Phi^{\prime}=\frac{e B S}{\hbar}+\varphi$ that varies with the magnetic field $B$ or the width of the mesa $W$ (affecting the area $S$ ). Similarly to the MZI at bulk FF 1 , the variation of the width induces an exponential damping in the transmission amplitude due to the non simultaneous arrival of the two WPs at the second BS. However, in this geometry, the length scale $\Sigma$ does not directly depend on geometrical parameters of the BS, but on the dynamics of the WPs in the phase shifter, due to the different velocities of the two channels in the phase shifter.

Regarding the magnetically-driven $\mathrm{AB}$ oscillations, numerical data (dots) in figure 10(a) show that the transmission coefficients slightly increase with $\mathrm{B}$, while the theoretical model (solid line) predicts a constant visibility. Indeed, the latter does not take into account the magnetic dependence of the $\mathrm{BS}$, and that the transmission coefficients of the phase shifter do not actually have an unitary module. Support 
simulation in [69] prove indeed that an increase of the magnetic field increases the scattering from the first to the second channel at the BS. Additionally, when B decreases, the LLs reduce their energy resolution, thus favoring interchannel scattering. The phase shifter acts therefore as an additional BS that decreases the visibility of the device.

In this geometry, the visibility reaches the value vis $=0.87$ for a Gaussian WP with $\sigma=60 \mathrm{~nm}$ and $W=W_{0}$. Higher values are predicted for experimental implementations, where single-electron WPs are usually injected with smaller energy resolution.

The possibility of implementing a high-performance MZI, should be feasible with current nanotechnology, with a scalable geometry and a small loop area making this device an ideal candidate for the engineering of two-qubit operations, as described in the following section.

\section{Two-particle dynamics}

\subsection{Hong-Ou-Mandel interferometer}

In the HOM interferometer two indistinguishable particles [118], generated from independent sources, interact at a halfreflecting BS and are recollected at two detectors, where the two-particle statistics is tested by measuring the coincidence counts. In optics, two identical photons are emitted from synchronized sources and then simultaneously detected at the same output due to their bosonic nature, so that the bunching probability is 1 . In an experimental realization of the device, this translates into a full peak in the coincidence counts. By reducing the synchronization of the two sources, i.e. by a time shift $\Delta t$, the bunching probability tends to $1 / 2$ in the limit $\Delta t \rightarrow \infty$, as expected for distinguishable particles [119].

In the electronic counterpart of the HOM experiment, a perfect antibunching of the two charges is predicted, owing to their fermionic nature. In fact, Pauli exclusion principle prevents that two indistinguishable electrons occupy simultaneously the same output. In real experiments, however, the measurement of the coincidence counts would require singleshot detection of flying electrons in a semiconductor device, which is not acheavable yet. Differently from the photon case, here the bunching probability is recovered from low-frequency current noise in the output currents [120]. The earliest measurements on electron antibunching were performed by injecting a continuous current of electrons in a HOM [121] and HBT interferometer [8, 49], but the use of electron beams prevents the interference to be directly interpreted as the overlap between single-electron WPs. In 2013, Bocquillon et al performed the first $\mathrm{HOM}$ experiment in the $\mathrm{IQH}$ regime with single-electron sources [24]: two mesoscopic capacitors inject an electron followed by a hole in anti-chiral edge channels, and the excess low frequency noise is measured at one of the outputs at different synchronization times $\Delta t$. This measurement exposes the so called (Pauli dip) in the antibunching counts. In this geometry, the electron BS is a QPC, whose action on the electron state is detailed in section 4.3. The presence of a minimum in the excess noise as a function of $\Delta t$ proves the generation of coherent and indistinguishable particles from independent sources. Interestingly, the minimum of the Pauli dip is not zero, differently from the theoretical predictions of stationary models.

This apparent violation of the Pauli exclusion principle occurs also in later experiments at bulk FF 2 [13, 76], and it has been related to the nature of Landau excitations, which are injected as single-electron WPs by mesoscopic capacitors $[72,81]$. These sources generate linear combinations of edgemagnetoplasmon excitations, whose coherence is destroyed by Coulomb inter-channel and intra-channel interactions. At bulk FF 2, after the two edge channels run parallel for a long enough distance $L_{\text {frac }}=\frac{\hbar v_{g}}{\sigma}$, the charge of the single-electron pulse is split between the two LLs. This source of decoherence, known as charge fractionalization and already observed in single-electron devices [7, 122, 123], quenches the effect of exchange-symmetry in the two-electron antibunching, thus increasing the minimum value of the Pauli dip for larger WPs [80]. It is however possible to prevent this exotic effect by increasing the lateral distance between the two channels with a local variation of the FF [69], or by looping the inner one [124].

On the other side, time-dependent numerical simulations show that the non-zero Pauli dip can also be intrinsically related to the localized nature of the electron state $[65,88]$. Indeed, a steady state picture for ESs-widely adopted in literature-does not fully capture the features of two-electron [125] antibunching in a HOM interferometer. Marian et al [65] investigate two-electron tunneling on a barrier in a timedependent framework [126], and provide the bunching probability for an exchange and Coulomb-driven scattering [127]. This 1D framework is equivalent to a HOM setup, with the electrons initialized in quasi-particle WPs with opposite central $k_{0}$. By using a time-dependent algorithm, all the energy-related information about the system are automatically accounted for, and no effective guesses must be done to include the energy selectivity of the barrier. The energies of the system are set to split each electron WP with 50\% of integrated transmission probability. However, similarly to a real QPC, the highest energy components are mainly transmitted, while the lowest are reflected. This means that non identical reflected and transmitted WPs are generated after the scattering at the BS. Since only WPs with identical $k$ components at the same side of the device are canceled by exchange-symmetry, the residual non zero overlap contributes to a residual bunching probability. The stationary behavior is restored only in the limit $\sigma \rightarrow \infty$, where the WP is well approximated by a plane wave. This trend differs from the measured one in presence of charge fractionalization, which remarks the difference between the two scenarios.

A full-scale 2D simulation of the HOM interferometer was presented in [88], thus overcoming the limits of the 1D effective geometry. Here, the external potential profile is shaped to reproduce a realistic QPC, and the electrons are initialized in single-electron Gaussian WPs of the exact ESs (figure 11(a)). The energies are set to simulate a simple case of bulk FF 1, so that interchannel interaction are not expected 

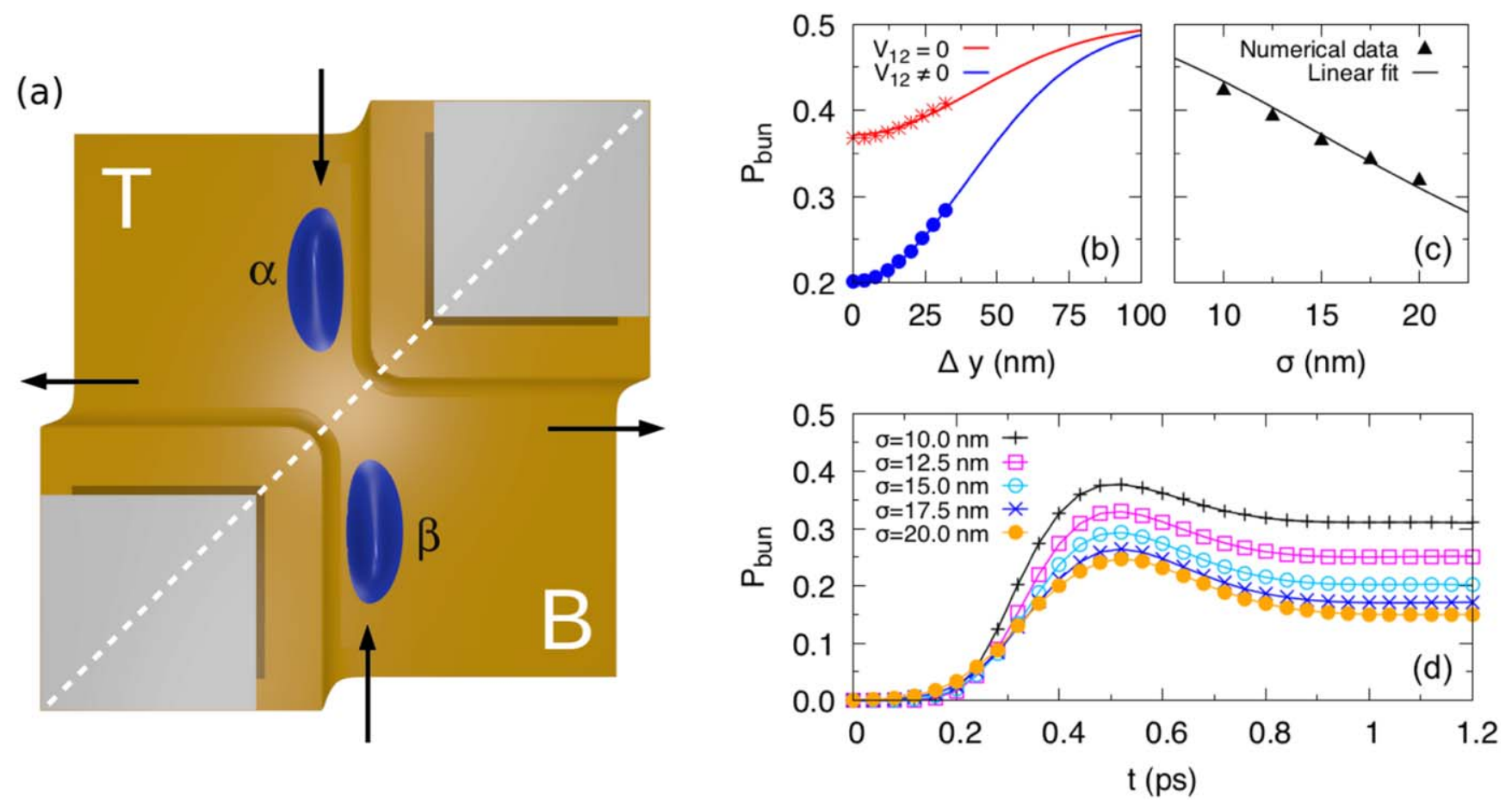

Figure 11. (a) Top-view of the external potential profile (gold region) generated by top gates in a realistic HOM interferometer and initial single-electron Gaussian WPs (blue shapes). (b) Stationary bunching probability induced by the exchange symmetry, as a function of the inital offset in the $y$ position of the two carriers, equivalent to a variation of the synchronization time. Red stars and blue dots correspond to the case without and with Coulomb interaction, respectively. (c) Stationary bunching probability for different WP real-space dispersions in presence of exchange symmetry alone. (d) Time evolution of the bunching probability for different WP sizes. Figures are adapted from [88].

to be effective. Thanks to the use of the time-dependent algorithm described in section 5.1, numerical results in this scenario do not require any effective model for the transmission probability. Rather, it is automatically accounted for exactly by the definition of the external potential profile.

If, on one hand, the dynamical bunching probability

$P_{b}(t)=\int_{S_{\mathrm{TT}}}|\Psi|^{2} \mathrm{~d} x_{1} \mathrm{~d} y_{1} \mathrm{~d} x_{2} \mathrm{~d} y_{2}+\int_{S_{\mathrm{BB}}}|\Psi|^{2} \mathrm{~d} x_{1} \mathrm{~d} y_{1} \mathrm{~d} x_{2} \mathrm{~d} y_{2}$,

which is computed with the exact two-particle wave function $\Psi\left(x_{1}, y_{1}, x_{2}, y_{2}\right)$, requires the exploitation of high performance computing resources (section 5.1), on the other hand, an approximated analytical formula in stationary conditions can be obtained by using the empirical transmission coefficients of a realistic 2D QPC given in equation (35). The two-electron wave function

$$
|\Psi\rangle=\frac{\left|\phi_{\alpha}\right\rangle\left|\phi_{\beta}\right\rangle-\left|\phi_{\beta}\right\rangle\left|\phi_{\alpha}\right\rangle}{\sqrt{2}}
$$

is obtained from single-electron WPs $\left|\phi_{\alpha(\beta)}\right\rangle$ injected from independent sources in opposite edge channels. The BS generates a reflected $\left|\phi_{\alpha(\beta)}^{R}\right\rangle$ and a transmitted component $\left|\phi_{\alpha(\beta)}^{T}\right\rangle$ that are not identical, according to the findings of [103] (where the QPC is integrated in a MZI) and of [65] (simulating a 1D effective model). The well-known bunching probability

$P_{\text {bun }}=\left\langle\phi_{\alpha}^{R} \mid \phi_{\alpha}^{R}\right\rangle\left\langle\phi_{\beta}^{T} \mid \phi_{\beta} T\right\rangle+\left\langle\phi_{\alpha}^{T} \mid \phi_{\alpha}^{T}\right\rangle\left\langle\phi_{\beta}^{R} \mid \phi_{\beta}^{R}\right\rangle-2\left|\left\langle\phi_{\alpha}^{T} \mid \phi_{\beta}^{R}\right\rangle\right|^{2}$

then reads, for a WP in the 2D geometry,

$$
P_{\text {bun }}=\frac{1}{2}-\frac{1}{2} \frac{\sigma^{2}}{\Sigma^{2}} \mathrm{e}^{\frac{-\Delta y^{2}}{4 \Sigma^{2}}},
$$

with $\Sigma^{2}=\sigma^{2}+\alpha^{2} / 8 \gamma$ (figure 11(b)). This effective length $\Sigma$ clarifies the relation between the spatial dispersion of the WP $\sigma$ and the geometry of the QPC, encoded in the geometrical parameters $\alpha$ and $\gamma$. Equation (71) predicts a non-zero Pauli dip regardless the type of excitation generated by the two independent sources, and defines the regime $\left(\sigma^{2} \gg \alpha^{2} / 8 \gamma\right)$ where the HOM acts as a probe for the indistinguishability of particles generated from independent sources. $\Sigma$ defines also the length scale for the suppression of two-particle interference in the HOM interferometer.

Moreover, thanks to the computation of the exact fourdegrees-of-freedom wave function for a two-electron system, the Coulomb interaction between the two charges can be simulated exactly in a realistic geometry. Note, indeed, that in the $2 \mathrm{D}$ real space the effect of electron repulsion is expected to differ from the same in 1D systems [128, 129]. In the latter case, the two electrons are confined on the same rail, e.g. in the $\hat{x}$-direction, and they are forced to get across $x_{1}=x_{2}$, thus feeling an effective infinite barrier during the scattering for $d=0$. On the contrary, in presence of the exact Coulomb 


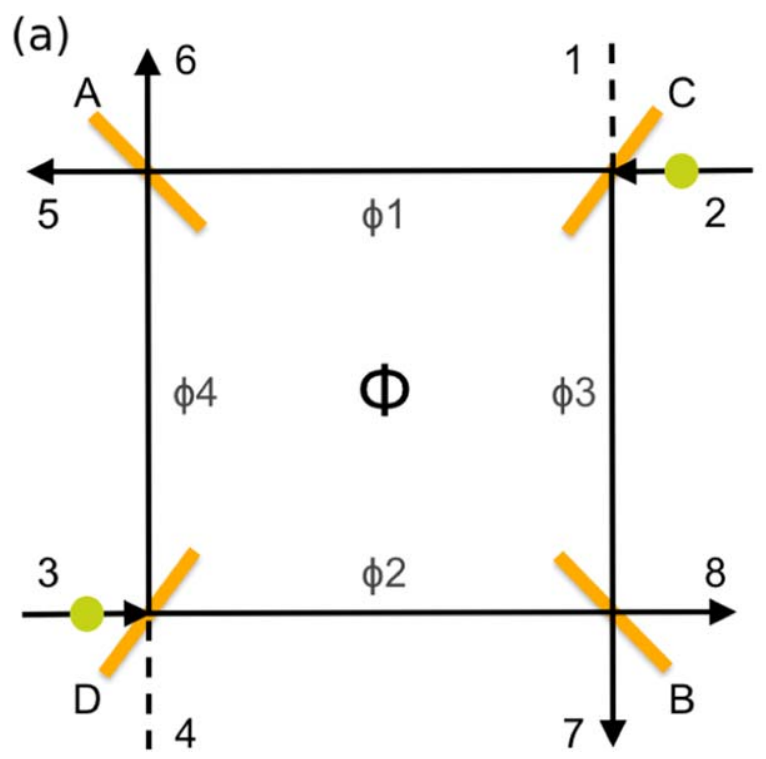

(b)

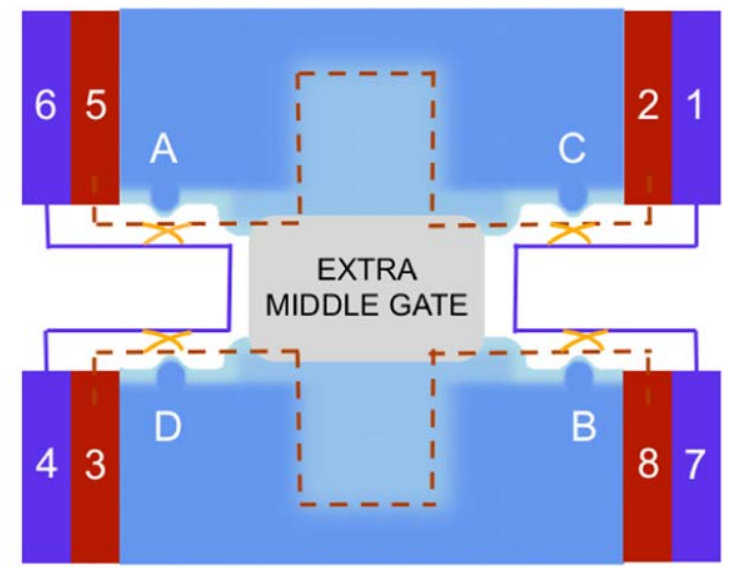

Figure 12. (a) General scheme of an optical HBT interferometer. Particles are injected at contacts 2 and 3 and collected at $\alpha=5,6$ and $\beta=7$, 8. (b) HBT interferometer proposed by Giovannetti et al implemented in a IQH device at bulk FF 2 [97].

interaction between the two-particles

$V_{12}\left(x_{1}, y_{1}, x_{2}, y_{2}\right)=\frac{e^{2}}{4 \pi \epsilon \sqrt{\left(x_{1}-x_{2}\right)^{2}+\left(y_{1}-y_{2}\right)^{2}+d^{2}}}$,

the bunching probability is not cancelled at $d=0$, where $d$ accounts for the finite thickness of the 2D system in the divergence at $x_{1}\left(y_{1}\right)=x_{2}\left(y_{2}\right)$ [88]. This explains why each charge is only partially reflected by the interparticle Coulomb potential. Furthermore, in presence of Coulomb repulsion, $P_{\text {bun }}$ does not increase linearly with increasing $\sigma$ (figure 11(c)), but rather it saturates (figure 11(d)).

This trend characterizes also the spatial entanglement [130] between the real-space distributions of the antibunched electrons, as reported in [88]. By dividing the device in two separated regions, labeled as top $(T)$ and bottom $(B)$ in figure 11(a), the reduced density matrix

$\rho_{T}\left(x_{1}, x_{1}^{\prime}\right)=\int_{x_{2} \in B} \mathrm{~d} x_{2} \Psi\left(x_{1}, y_{1}^{*}, x_{2}, y_{2}^{*}\right) \Psi^{*}\left(x_{2}, y_{2}^{*}, x_{1}^{\prime}, y_{1}^{*}\right)$,

can be computed from the exact two-electron wave function $\Psi$. Here, the chirality of the edge channels allows one to project the two-electron state on the $1 \mathrm{D}$ rails at fixed coordinates $y_{1}^{*} \in T$ and $y_{2}^{*} \in B$, so that only one of the possible antibunched configurations of the two-electron system is considered. The von Neumann entropy $S=\operatorname{Tr}\left[\rho_{T} \ln \left(\rho_{T}\right)\right]$, which provides the degree of entanglement between the particles in the two regions, is shown to decrease with increasing spatial distribution of the WPs, as well as by introducing Coulomb repulsion between the carriers.

\subsection{HBT interferometer}

The HOM interferometer described in the previous section is a viable platform for testing many-body statistics. Moreover, it represents a building-block for the implementation of the HBT experiment, which has the potential to detect and control the degree of entanglement between particles generated from uncorrelated sources. In its first realization [131], the angular diameter of the binary star Sirius was determined by measuring the interference between light signals from different portions of the system. Driven by the many-body statistics of indistinguishable photons, in a HBT geometry the interference pattern is not present in the intensity of the signal measured at a single detector, but it is only visible in intensity-intensity correlations between signals from uncorrelated detectors. Because of exchange-symmetry, the HBT effect is achievable also in the intensity correlation of electron beams. However, if an optic HBT interferometer has been technologically feasible with field-emission into vacuum, the electron analog requires a solid-state implementation in electrical conductors, where the Fermi gas is degenerate at low temperatures [132]. The long-range coherence of ESs in the IQH regime is then the ideal playground to observe a high-visibility interference pattern, but, differently from photon-based devices, coincidence counts can not be clearly detected in electronic devices. Intensity-intensity correlations are so defined through the quantum partition noise and cross-correlations of current fluctuations [133, 134].

Figure 12(a) reports a scheme of the HBT interferometer, whose earliest investigation in the IQH regime is based on ESs at unitary bulk FF in a Corbino geometry [53, 135]. Two electron currents are generated at contact 2 and 3, while four QPCs, acting as electron BSs, partition the impinging beams towards four detectors. No single channel clearly encloses an AB flux, while the paths of currents at different detectors do. The zero-frequency cross correlations $S_{\alpha \beta}$ of the current fluctuations $\Delta I_{\alpha}$ and $\Delta I_{\beta}$ at detector $\alpha$ and $\beta$,

$$
S_{\alpha \beta}=\int \mathrm{d} t\left\langle\left[\Delta I_{\alpha}(t) \Delta I_{\beta}(0)+\Delta I_{\beta}(0) \Delta I_{\alpha}(t)\right]\right\rangle,
$$

can be rewritten in terms of the scattering matrix $s_{\alpha \beta}$, which 
relates the current incoming at contact $\beta$ to the outgoing from contact $\alpha$ [134]. By assuming electron currents entering the device from the reservoirs at contacts 2 and 3, and exiting through contacts $\alpha=5,6$ and $\beta=7,8, S_{\alpha \beta}$ reads

$$
S_{\alpha \beta}=-\frac{2 e^{2}}{h} \int \mathrm{d} E\left|s_{\alpha_{2}}^{*} s_{\beta_{2}}+s_{\alpha_{3}}^{*} s_{\beta_{3}}\right|^{2}\left(f-f_{0}\right)^{2},
$$

with $f$ Fermi distribution function of the emitting reservoirs and $f_{0}$ grounded distribution function of the remaining contacts. In the present scenario, phases are added to the scattering matrices as multiplicative terms, thus not leading to interference effects in single currents. Current intensities at the grounded contacts read:

$$
I_{5(7)}=I_{6(8)}=\frac{2 e^{2}}{h} T(R) V
$$

with $T$ and $R$ transmission (reflection) and reflection (transmission) coefficients of the QPC partitioning the signal from contact 2 (3). A phase dependence arises in the current-current correlations, as for example

$$
S_{5,8}=-\frac{e^{2}}{4 h}|e V|\left(1+\cos \left(\phi_{1}+\phi_{2}-\phi_{3}-\phi_{4}\right)\right),
$$

with $T=R=1 / 2$. The four channels enclose then an $\mathrm{AB}$ flux that contributes positively for $\phi_{1}, \phi_{2}$ and negatively for $\phi_{3}, \phi_{4}$, so that the total phase in equation (77) is rewritten as $\phi_{0}=\phi_{1}+\phi_{2}-\phi_{3}-\phi_{4}+2 \Phi / \Phi_{0}$, with $2 \Phi / \Phi_{0}=\oint A \cdot \mathrm{d} l$ and $A$ magnetic vector potential. This makes $S_{5,8}$ sensitive to variation of the $\mathrm{AB}$ flux, as proved experimentally in [8]. In this work, instead of implementing the looped Corbino geometry proposed in [53], two single-channel MZI [22] in the IQH regime are connected via a tunable QPC. As predicted theoretically, its opening suppresses $\mathrm{AB}$ oscillations in the current amplitude of each MZI, while the interference pattern is recovered in the cross-correlations spectral density. This experimentally proves that the HBT effect is exclusively driven by two-particle exchange symmetry.

The observation of an interference pattern driven by electron antibunching has been interpreted as a signature of entanglement, to be detected with a violation of Bell's inequality (BI) [136]. BI, originally expressed with the joint detection probabilities of two particles at uncorrelated detectors, must then be reformulated in terms of the zero-frequency cross correlations [137]. By assuming strongly asymmetric BSs $(R \ll 1$ at $\mathrm{C}$ and D in figure 12$)$, it is possible to achieve a regime with no counterpart in optics: the HBT interferometer is fed by electron-hole pairs generated from uncorrelated sources [53, 135]. Within this condition, the vacuum is noiseless and the coherence time $\tau_{C}=\frac{h}{e V}$ is smaller than the emission time $\tau_{e}=\frac{h}{e V R}$ of the electron-hole pairs, so that the zero-frequency cross-correlation provides the same information of coincidence counts over a long time. To observe the interference pattern, the phases in equation (77) are typically varied by tuning the transmission coefficients of the BSs before the detector contacts (e.g. A and $B$ in figure 12(a)). Moreover, [53] proves that orbital entanglement, i.e. the one related to the spatial degrees of freedom, can be detected via a violation of BI not only for electronhole pairs, but also for pairs of electrons.

In the experiments described above, the simultaneous detection of the two electrons can be considered as a postselective measurement of the orbitally-entangled pair of electrons. Indeed, if two electrons are simultaneously detected at two different contacts, they must have been injected from different reservoirs due to their antibunching at the QPCs, as simulated in section 6.1. Since the paths of these two electrons can not be distinguished, the two particles must be orbitally entangled. However, the two charges are generated from different sources, and the state emitted from these contacts is described by a product state with respect to the single electrons. This is of course not valid for a photon-based HBT interferometer, where particles bunch.

Finally, we note that strong dephasing reduces the orbital entanglement between the two particles, as well as the visibility of the interference pattern, but it is not expected to fully suppress it [96]. Indeed, it impacts the minimum temperature [138] to experimentally observe the violation of BI [139], which is precluded by a low visibility, as in the setup of [8].

The HBT geometries investigated above are jeopardized by the well-known topological limit of Hall interferometers at bulk FF 1, where Ohmic contacts are inserted in the loop areas and only one channel is available at the output of each MZI (section 4). Reference [97] proposes an alternative geometry based on the concatenation of two multi-channel MZI at bulk FF 2 (section 5.2). As shown in figure 12(b), the two MZIs are connected in parallel, and an extra middle gate is introduced in the loop areas to increase the local FF to 1. With respect to the geometry of the MZI simulated in section 5.2, the middle gate diverts the path of the inner channel $(n=1)$, which reaches one of the detectors on the opposite edge of the 2DEG. The potentially smaller extension of this central middle gate, whose engineering has been described in section 4.2, can in principle reduce the sensitivity of the HBT to fluctuations of the AB flux in the two-electron interference, and lead to experimentally prove the violation of BI.

\section{Summary and conclusions}

The search for quantum computing devices is nowadays a prominent research effort, expecially in the field of semiconductor nanoelectronics. Moreover, quantum information processing framework and entanglement theory have an important role also in the assessment of coherent dynamics beyond computer science and physics [140]. The guideline of this work is to show, through the review of recent proposals, that it is possible to devise semiconductor-based quantum gates suitable to process quantum information, by resorting to coherent carrier transport in Hall ESs. Indeed, carriers propagating in such channels are topologically protected from decoherence phenomena and serial operations of quantum gates can be realized, as long as the coherent dynamics of carriers is preserved. Devices operating in the IQH regime, where a magnetic field and a suitable potential landscape 
create specific paths of quasi-1D edge channels, has been simulated numerically, and quantum interference patterns have been assessed. It has been shown theoretically that this flying-qubit implementation of quantum computing architecture is able to realize a universal set of quantum gates.

We have considered the injection of single strongly localized carriers in ESs, and compared it with the case of a current of delocalized electrons. In terms of quantum-information processing, the realization of qubits is simpler if resorting to localized carriers, since the qubit state can be described by the spin state, the Landau index or the spatial localization of the carrier itself. Specifically, we have focused on a qubit encoding based either on the which-path localizetion, or on the LL index.

There are three available protocols for the injection of single carriers in ESs. The so called 'Leviton' (a minimal excitation of the Fermi sea), the mesoscopic capacitors (realized by means of a dynamical coupling of the source with the device ESs) and quantum dot pumps (based on tunable barriers allowing confined electrons to be controllably injected in the device). For the latter method a theoretical protocol has been proposed to generate and detect almost identical Gaussian WPs from non-identical quantum dot pumps [84]. The method adopted in this work for the numerical propagation of the WP does not rely on a specific form of the initial state. In the numerical simulation reported here we consider a minimum uncertainty WP as the one that is generated with quantum dot pumps.

We note that electron transport in the IQH regime is usually studied by means of 1D analytical models. Here, we have addressed the problem in a full-scale 2D geometry, through a numerical solver of the time-dependent Schrödinger equation able to provide an exact solution of the single-particle and two-particle dynamics of WPs in ESs. In fact, timedependent modeling of few-particle systems is an ideal framework to expose the building up of quantum correlations $[141,142]$. With such an approach it is easy to include electron-electron interaction or time-dependent potentials in the system, and to assess the dynamical properties of the twoparticle WP. Also, we showed that, by suitably designing the 2DEG potential profile, an entangling two-qubit gate can be realized by exploiting Coulomb interaction, a fundamental element for the universal set of quantum gates.

The visibility of single-electron MZIs, where the qubit is either defined by carrier position or Landau index, has been obtained, and the effect of the energy selectivity of BSs has been quantified. The specific implementation of the MZI operating at FF 2 described in section 5.2 solves the scalability problems of the MZI at FF 1, thus potentially enabling the concatenation in series of multiple devices and their integration into complex quantum computing architectures.

As a second viable platform for realize a flying qubit, a proposal for an electronic HOM interferometer operating in the IQH regime has been discussed and simulated. The system operates at FF 1 and the qubit is defined by the spatial localization of the WPs describing the carriers. Here, the longrange $2 \mathrm{D}$ Coulomb interaction has been included, and the interplay between Coulomb repulsion and exchange symmetry has been investigated.

\section{Acknowledgments}

We thank Andrea Beggi, Chiara Galeotti and Xavier Oriols for fruitful discussions. We acknowledge CINECA for HPC resources and support under the ISCRA initiative (DYNAMET-HP10C1MI91 and QUPIDO-HP10CPRABZ). The numerical simulations have been performed under the Project HPC-EUROPA3 (INFRAIA-2016-1-730897), with the support of the EC Research Innovation Action under the H2020 Programme. LB gratefully acknowledges the support of Departament d'Enginyeria Electrònica (UAB) and the computer resources and technical support provided by Barcelona Supercomputing Center (project HPC17D8XLY).

\section{ORCID iDs}

Paolo Bordone (10 https://orcid.org/0000-0002-4313-0732

Laura Bellentani (i) https://orcid.org/0000-0002-8048-0088

Andrea Bertoni (ib https://orcid.org/0000-0003-3103-7239

\section{References}

[1] Ramamoorthy A, Bird J P and Reno J L 2007 Using split-gate structures to explore the implementation of a coupledelectron-waveguide qubit scheme J. Phys. Condens. Matter 19276205

[2] Yamamoto M, Takada S, Bauerle C, Watanabe K, Wieck A D and Tarucha S 2012 Electrical control of a solidstate flying qubit Nat. Nanotechnol. 7247

[3] Büttiker M 1988 Absence of backscattering in the quantum Hall effect in multiprobe conductors Phys. Rev. B 38 9375-89

[4] van Wees B J, Kouwenhoven L P, Harmans C J P M, Williamson J G, Timmering C E, Broekaart M E I, Foxon C T and Harris J J 1989 Observation of zerodimensional states in a one-dimensional electron interferometer Phys. Rev. Lett. 62 2523-6

[5] Bird J P, Ishibashi K, Stopa M, Aoyagi Y and Sugano T 1994 Coulomb blockade of the Aharonov-Bohm effect in GaAs/ $\mathrm{Al}_{x} \mathrm{Ga}_{1-x}$ As quantum dots Phys. Rev. B 50 14983-90

[6] Roulleau P, Portier F, Roche P, Cavanna A, Faini G, Gennser U and Mailly D 2008 Direct measurement of the coherence length of edge states in the integer quantum Hall regime Phys. Rev. Lett. 100126802

[7] Neder I, Heiblum M, Levinson Y, Mahalu D and Umansky V 2006 Unexpected behavior in a two-path electron interferometer Phys. Rev. Lett. 96016804

[8] Neder I, Ofek N, Chung Y, Heiblum M, Mahalu D and Umansky V 2007 Interference between two indistinguishable electrons from independent sources Nature 448333

[9] Levitov L S, Lee H and Lesovik G B 1996 Electron counting statistics and coherent states of electric current J. Math. Phys. 37 4845-66

[10] Mahé A, Parmentier F D, Bocquillon E, Berroir J-M, Glattli D C, Kontos T, Plaçais B, Fève G, Cavanna A and 
Jin Y 2010 Current correlations of an on-demand singleelectron emitter Phys. Rev. B 82201309

[11] Devyatov E V 2013 Electronic interferometers in the quantum Hall effect regime Low Temp. Phys. 39 7-17

[12] Rech J, Ferraro D, Jonckheere T, Vannucci L, Sassetti M and Martin T 2017 Minimal excitations in the fractional quantum Hall regime Phys. Rev. Lett. 118076801

[13] Bocquillon E, Freulon V, Berroir J-M, Degiovanni P, Plaçais B, Cavanna A, Jin Y and Fève G 2013 Separation of neutral and charge modes in one-dimensional chiral edge channels Nat. Commun. 411839

[14] Bauerle C, Glattli D C, Meunier T, Portier F, Roche P, Roulleau P, Takada S and Waintal X 2018 Coherent control of single electrons: a review of current progress Rep. Prog. Phys. 81056503

[15] Bertoni A, Bordone P, Brunetti R, Jacoboni C and Reggiani S 2000 Quantum logic gates based on coherent electron transport in quantum wires Phys. Rev. Lett. 84 5912-5

[16] Ionicioiu R, Amaratunga G and Udrea F 2001 Quantum computation with ballistic electrons Int. J. Mod. Phys. B 15 $125-33$

[17] Ramamoorthy A, Bird J P and Reno J L 2006 Quantum asymmetry of switching in laterally coupled quantum wires with tunable coupling strength Appl. Phys. Lett. 89153128

[18] Ramamoorthy A, Akis R and Bird J P 2006 Influence of realistic potential profile of coupled electron waveguide on electron switching characteristics IEEE Trans. Nanotechnol. $5712-5$

[19] Aharony A, Takada S, Entin-Wohlman O, Yamamoto M and Tarucha S 2014 Aharonov-Bohm interferometry with a tunnel-coupled wire New J. Phys. 16083015

[20] Abdullah N R 2016 Magnetically and photonically tunable double waveguide inverter IEEE J. Quantum Electron. 52 $1-6$

[21] Olendski O 2018 Electric-field control of bound states and optical spectrum in window-coupled quantum waveguides J. Appl. Phys. 124095704

[22] Weisz E, Choi H K, Sivan I, Heiblum M, Gefen Y, Mahalu D and Umansky V 2014 An electronic quantum eraser Science 344 1363-6

[23] Metwally N, Abdelaty M and Obada A-S F 2005 Entangled states and information induced by the atom-field interaction Opt. Commun. 250 148-56

[24] Bocquillon E, Freulon V, Berroir J-M, Degiovanni P, Plaçais B, Cavanna A, Jin Y and Fève G 2013 Coherence and indistinguishability of single electrons emitted by independent sources Science 339 1054-7

[25] Obada A-S F, Abo-Kahla D A M, Metwally N and Abdel-Aty M 2011 The quantum computational speed of a single cooper-pair box Physica E 43 1792-7

[26] Abdel-Aty M 2004 Perspectives for a mixed two-qubit system with binomial quantum states J. Opt. B: Quantum Semiclass. Opt. 6 201-10

[27] Bertoni A 2007 Perspectives on solid-state flying qubits J. Comput. Electron. 6 67-72

[28] Bertoni A 2009 Charge-Based Solid-State Flying Qubits (New York, NY: Springer) pp 1011-27

[29] Ciccarello F, Browne D E, Kwek L C, Schomerus H, Zarcone M and Bose S 2012 Quasideterministic realization of a universal quantum gate in a single scattering process Phys. Rev. A 85050305

[30] Marzolino U and Buchleitner A 2015 Quantum teleportation with identical particles Phys. Rev. A 91032316

[31] Debnath S, Linke N M, Figgatt C, Landsman K A, Wright K and Monroe C 2016 Demonstration of a small programmable quantum computer with atomic qubits Nature 536 63-6
[32] Wendin G 2017 Quantum information processing with superconducting circuits: a review Rep. Prog. Phys. 80 106001

[33] Bonen S et al 2019 Cryogenic characterization of 22-nm FDSOI CMOS technology for quantum computing ICs IEEE Electron Device Lett. 40 127-30

[34] Horodecki R, Horodecki P, Horodecki M and Horodecki K 2009 Quantum entanglement Rev. Mod. Phys. 81 865-942

[35] Simonović N S and Nazmitdinov R G 2015 Magnetic alteration of entanglement in two-electron quantum dots Phys. Rev. A 92052332

[36] Kenfack L T, Tchoffo M and Fai L C 2017 Dynamics of tripartite quantum entanglement and discord under a classical dephasing random telegraph noise Eur. Phys. J. Plus 13291

[37] Arthur T T, Martin T and Fai L C 2017 Quantum correlations and coherence dynamics in qutrit-qutrit systems under mixed classical environmental noises Int. J. Quantum Inf. 15 1750047

[38] Tsokeng A T, Tchoffo M and Fai L C 2017 Quantum correlations and decoherence dynamics for a qutrit-qutrit system under random telegraph noise Quantum Inf. Process. 16191

[39] Yang J, Mu Q-X and Huang Y-X 2018 The dynamics of tripartite quantum correlations under Ornstein-Uhlenbeck noise Mod. Phys. Lett. B 321850381

[40] Arthur T T, Martin T and Fai L C 2018 Dynamics of tripartite quantum correlations and decoherence in flux qubit systems under local and non-local static noise Quantum Inf. Process. 17136

[41] Ellerman D 2018 Logical entropy: introduction to classical and quantum logical information theory Entropy 20679

[42] Buscemi F, Bordone P and Bertoni A 2008 Effects of scattering resonances on carrier-carrier entanglement in charged quantum dots J. Comput. Electron. 7 263-7

[43] Gollisch H, Giebels F and Feder R 2019 Evolution of entanglement in electron-electron collisions J. Electron. Spectrosc. Relat. Phenom. 232 83-8

[44] Klitzing K V, Dorda G and Pepper M 1980 New method for high-accuracy determination of the fine-structure constant based on quantized Hall resistance Phys. Rev. Lett. 45 494-7

[45] Ferry D K, Goodnick S M and Bird J 2009 Transport in Nanostructures vol 6 2nd edn (Cambridge: Cambridge University Press) (https://doi.org/10.1017/ CBO9780511840463)

[46] Montambaux G 2011 Semiclassical quantization of skipping orbits Eur. Phys. J. B 79 215-24

[47] Jiang W-Y, Zhao Z-M, Yang H, Zheng L and Zheng T-Y 2013 Quantum dynamics of atomic spatial decoherence in a squeezed vacuum field Int. J. Theor. Phys. 524008

[48] Puviani M, Manghi F and Bertoni A 2017 Dynamics and control of edge states in laser-driven graphene nanoribbons Phys. Rev. B 95235430

[49] Oliver W D, Kim J, Liu R C and Yamamoto Y 1999 Hanbury Brown and twiss-type experiment with electrons Science 284 299-301

[50] Henny M, Oberholzer S, Strunk C, Heinzel T, Ensslin K, Holland M and Schönenberger C 1999 The fermionic Hanbury Brown and Twiss experiment Science 284 296-8

[51] Ji Y, Chung Y, Sprinzak D, Heiblum M, Mahalu D and Shtrikman H 2003 An electronic Mach-Zehnder interferometer Nature $\mathbf{4 2 2} 415$

[52] Karmakar B, Venturelli D, Chirolli L, Giovannetti V, Fazio R, Roddaro S, Pfeiffer L N, West K W, Taddei F and Pellegrini V 2015 Nanoscale Mach-Zehnder interferometer with spin-resolved quantum Hall edge states Phys. Rev. B 92 195303

[53] Samuelsson P, Sukhorukov E V and Büttiker M 2004 Twoparticle Aharonov-Bohm effect and entanglement in the 
electronic Hanbury Brown-Twiss setup Phys. Rev. Lett. 92 026805

[54] Kang K 2007 Electronic Mach-Zehnder quantum eraser Phys. Rev. B 75125326

[55] Chirolli L, Taddei F, Fazio R and Giovannetti V 2013 Interactions in electronic Mach-Zehnder interferometers with copropagating edge channels Phys. Rev. Lett. 111 036801

[56] Radu A, Iftimie S and Dragoman D 2019 Ballistic 3-port interferometric logic gates in the quantum Hall regime Physica E 109 144-51

[57] Fève G, Mahé A, Berroir J-M, Kontos T, Plaçais B, Glattli D C, Cavanna A, Etienne B and Jin Y 2007 An ondemand coherent single-electron source Science $\mathbf{3 1 6}$ 1169-72

[58] Kaestner B et al 2008 Single-parameter nonadiabatic quantized charge pumping Phys. Rev. B 77153301

[59] Fletcher J D et al 2012 Stabilization of single-electron pumps by high magnetic fields Phys. Rev. B 86155311

[60] Dubois J, Jullien T, Portier F, Roche P, Cavanna A, Jin Y, Wegscheider W, Roulleau P and Glattli D C 2013 Minimalexcitation states for electron quantum optics using levitons Nature $\mathbf{5 0 2} 659$

[61] Kataoka M, Fletcher J D and Johnson N 2017 Time-resolved single-electron wave-packet detection Phys. Status Solidi b 2541600547

[62] Haack G, Moskalets M, Splettstoesser J and Büttiker M 2011 Coherence of single-electron sources from Mach-Zehnder interferometry Phys. Rev. B 84081303

[63] Keeling J, Klich I and Levitov L S 2006 Minimal excitation states of electrons in one-dimensional wires Phys. Rev. Lett. 97116403

[64] Keeling J, Shytov A V and Levitov L S 2008 Coherent particle transfer in an on-demand single-electron source Phys. Rev. Lett. 101196404

[65] Marian D, Colomés E and Oriols X 2015 Time-dependent exchange and tunneling: detection at the same place of two electrons emitted simultaneously from different sources J. Phys. Condens. Matter 27245302

[66] Gaury B, Weston J and Waintal X 2014 Stopping electrons with radio-frequency pulses in the quantum Hall regime Phys. Rev. B 90161305

[67] Beggi A, Bertoni A and Bordone P 2015 Time-dependent modelling of single-electron interferometry with edge-states J. Phys.: Conf. Ser. 647012023

[68] Bellentani L, Beggi A, Bordone P and Bertoni A 2017 Dynamics of copropagating edge states in a multichannel Mach-Zender interferometer J. Phys.: Conf. Ser. 906 012027

[69] Bellentani L, Beggi A, Bordone P and Bertoni A 2018 Dynamics and Hall-edge-state mixing of localized electrons in a two-channel Mach-Zehnder interferometer Phys. Rev. B 97205419

[70] Ivanov D A, Lee H W and Levitov L S 1997 Coherent states of alternating current Phys. Rev. B 56 6839-50

[71] Glattli D C and Roulleau P 2018 Pseudorandom binary injection of levitons for electron quantum optics Phys. Rev. B 97125407

[72] Ferraro D, Roussel B, Cabart C, Thibierge E, Fève G, Grenier C and Degiovanni P 2014 Real-time decoherence of Landau and Levitov quasiparticles in quantum Hall edge channels Phys. Rev. Lett. 113166403

[73] Glattli D C and Roulleau P 2016 Hanbury-Brown Twiss noise correlation with time controlled quasi-particles in ballistic quantum conductors Physica E 76 216-22

[74] Gabelli J, Fève G, Berroir J-M, Plaçais B, Cavanna A, Etienne B, Jin Y and Glattli D C 2006 Violation of Kirchhoff's laws for a coherent RC circuit Science $\mathbf{3 1 3}$ 499-502
[75] Büttiker M, Thomas H and Prêtre A 1993 Mesoscopic capacitors Phys. Lett. A 180 364-9

[76] Freulon V, Marguerite A, Berroir J-M, Plaçais B, Cavanna A, Jin Y and Fève G 2015 Hong-Ou-Mandel experiment for temporal investigation of single-electron fractionalization Nat. Commun. 66854

[77] Ronetti F, Vannucci L, Ferraro D, Jonckheere T, Rech J, Martin T and Sassetti M 2018 Crystallization of Levitons in the fractional quantum Hall regime Phys. Rev. B 98075401

[78] Wahl C, Rech J, Jonckheere T and Martin T 2014 Interactions and charge fractionalization in an electronic Hong-OuMandel interferometer Phys. Rev. Lett. 112046802

[79] Ferraro D, Ronetti F, Vannucci L, Acciai M, Rech J, Jockheere T, Martin T and Sassetti M 2018 Hong-OuMandel characterization of multiply charged Levitons European Phys. J. Spec. Top. 227 1345-59

[80] Marguerite A et al 2016 Decoherence and relaxation of a single electron in a one-dimensional conductor Phys. Rev. B 94115311

[81] Wahl C, Rech J, Jonckheere T and Martin T 2014 Interactions and charge fractionalization in an electronic Hong-OuMandel interferometer Phys. Rev. Lett. 112046802

[82] Blumenthal M D, Kaestner B, Li L, Giblin S, Janssen T J B M, Pepper M, Anderson D, Jones G and Ritchie D A 2007 Gigahertz quantized charge pumping Nat. Phys. 3393 343-7

[83] Kaestner B and Kashcheyevs V 2015 Non-adiabatic quantized charge pumping with tunable-barrier quantum dots: a review of current progress Rep. Prog. Phys. $\mathbf{7 8}$ 103901

[84] Ryu S, Kataoka M and Sim H-S 2016 Ultrafast emission and detection of a single-electron Gaussian wave packet: a theoretical study Phys. Rev. Lett. 117146802

[85] Yin Y 2018 On-demand electron source with tunable energy distribution J. Phys. Condens. Matter 30285301

[86] Yin Y 2019 Quasiparticle states of on-demand coherent electron sources J. Phys. Condens. Matter 31245301

[87] Emary C, Clark L A, Kataoka M and Johnson N 2019 Energy relaxation in hot electron quantum optics via acoustic and optical phonon emission Phys. Rev. B 99045306

[88] Bellentani L, Bordone P, Oriols X and Bertoni A 2019 Coulomb and exchange interaction effects on the exact twoelectron dynamics in the Hong-Ou-Mandel interferometer based on Hall edge states Phys. Rev. B 99245415

[89] Buscemi F, Bordone P and Bertoni A 2012 Electron interference and entanglement in coupled 1d systems with noise Eur. Phys. J. D 66312

[90] Rodriquez R, Oi D K L, Kataoka M, Barnes C H W, Ohshima T and Ekert A K 2005 Surface-acoustic-wave single-electron interferometry Phys. Rev. B 72085329

[91] Buscemi F, Bordone P and Bertoni A 2009 Validity of the single-particle approach for electron transport in quantum wires assisted by surface acoustic waves J. Phys. Condens. Matter 21305303

[92] Lew Yan Voon L C and Willatzen M 2011 Electromechanical phenomena in semiconductor nanostructures J. Appl. Phys. 109031101

[93] Yahyaie I, Buchanan D A, Bridges G E, Thomson D J and Oliver D R 2012 High-resolution imaging of gigahertz polarization response arising from the interference of reflected surface acoustic waves IEEE Trans. Ultrason. Ferroelectr. Freq. Control 59 1212-8

[94] Buscemi F, Bordone P and Bertoni A 2010 Quantum teleportation of electrons in quantum wires with surface acoustic waves Phys. Rev. B 81045312

[95] Goussev A 2017 Rotating Gaussian wave packets in weak external potentials Phys. Rev. A 96013617

[96] Chung V S-W, Samuelsson P and Büttiker M 2005 Visibility of current and shot noise in electrical Mach-Zehnder and 
Hanbury Brown twiss interferometers Phys. Rev. B 72 125320

[97] Giovannetti V, Taddei F, Frustaglia D and Fazio R 2008 Multichannel architecture for electronic quantum Hall interferometry Phys. Rev. B 77155320

[98] Litvin L V, Tranitz H-P, Wegscheider W and Strunk C 2007 Decoherence and single electron charging in an electronic Mach-Zehnder interferometer Phys. Rev. B 75033315

[99] Camino F E, Zhou W and Goldman V J 2005 AharonovBohm superperiod in a Laughlin quasiparticle interferometer Phys. Rev. Lett. 95246802

[100] Deviatov E V and Lorke A 2008 Experimental realization of a Fabry-Perot-type interferometer by copropagating edge states in the quantum Hall regime Phys. Rev. B 77161302

[101] Deviatov E V, Marquardt B, Lorke A, Biasiol G and Sorba L 2009 Interference effects in transport across a single incompressible strip at the edge of the fractional quantum Hall system Phys. Rev. B 79125312

[102] Deviatov E V, Ganczarczyk A, Lorke A, Biasiol G and Sorba L 2011 Quantum Hall Mach-Zehnder interferometer far beyond equilibrium Phys. Rev. B 84235313

[103] Beggi A, Bordone P, Buscemi F and Bertoni A 2015 Timedependent simulation and analytical modelling of electronic Mach-Zehnder interferometry with edge-states wave packets J. Phys. Condens. Matter 27475301

[104] Palacios J J and Tejedor C 1992 Effects of geometry on edge states in magnetic fields: adiabatic and nonadiabatic behavior Phys. Rev. B 45 9059-64

[105] Deviatov E V, Dolgopolov V T and Würtz A 2004 Charge redistribution between cyclotron-resolved edge states at high imbalance J. Exp. Theor. Phys. Lett. 79 504-9

[106] Venturelli D, Giovannetti V, Taddei F, Fazio R, Feinberg D, Usaj G and Balseiro C A 2011 Edge channel mixing induced by potential steps in an integer quantum Hall system Phys. Rev. B 83075315

[107] Würtz A, Wildfeuer R, Lorke A, Deviatov E V and Dolgopolov V T 2002 Separately contacted edge states: a spectroscopic tool for the investigation of the quantum Hall effect Phys. Rev. B 65075303

[108] Deviatov E V and Lorke A 2008 Separately contacted edge states at high imbalance in the integer and fractional quantum Hall effect regime Phys. Status Solidi b 245 366-77

[109] Roulleau P, Portier F, Glattli D C, Roche P, Cavanna A, Faini G, Gennser U and Mailly D 2007 Finite bias visibility of the electronic Mach-Zehnder interferometer Phys. Rev. B 76161309

[110] Karmakar B et al 2011 Controlled coupling of spin-resolved quantum Hall edge states Phys. Rev. Lett. 107236804

[111] Kramer T, Heller E J and Parrott R E 2008 An efficient and accurate method to obtain the energy-dependent green function for general potentials J. Phys.: Conf. Ser. 99 012010

[112] Kramer T, Kreisbeck C and Krueckl V 2010 Wave packet approach to transport in mesoscopic systems Phys. Scr. 82 038101

[113] Grasselli F, Bertoni A and Goldoni G 2016 Time-dependent scattering of a composite particle: a local self-energy approach for internal excitations Phys. Rev. B 94125418

[114] Grasselli F, Bertoni A and Goldoni G 2018 Classical and quantum dynamics of indirect excitons driven by surface acoustic waves Phys. Rev. B 98165407

[115] Metavitsiadis A, Dillenschneider R and Eggert S 2014 Impurity entanglement through electron scattering in a magnetic field Phys. Rev. B 89155406

[116] Ballicchia M, Weinbub J and Nedjalkov M 2018 Electron evolution around a repulsive dopant in a quantum wire: coherence effects Nanoscale 10 23037-49
[117] Buscemi F, Cancellieri E, Bordone P, Bertoni A and Jacoboni C 2008 Electron decoherence in a semiconductor due to electron-phonon scattering Phys. Status Solidi c 552

[118] Franco R L and Compagno G 2018 Indistinguishability of elementary systems as a resource for quantum information processing Phys. Rev. Lett. 120240403

[119] Hong C K, Ou Z Y and Mandel L 1987 Measurement of subpicosecond time intervals between two photons by interference Phys. Rev. Lett. 59 2044-6

[120] Beenakker C and Schönenberger C 2003 Quantum shot noise Phys. Today 5637

[121] Liu R C, Odom B, Yamamoto Y and Tarucha S 1998 Quantum interference in electron collision Nature 391 263-5 1

[122] Huynh P-A, Portier F, le Sueur H, Faini G, Gennser U, Mailly D, Pierre F, Wegscheider W and Roche P 2012 Quantum coherence engineering in the integer quantum Hall regime Phys. Rev. Lett. 108256802

[123] Helzel A, Litvin L V, Levkivskyi I P, Sukhorukov E V, Wegscheider W and Strunk C 2015 Counting statistics and dephasing transition in an electronic Mach-Zehnder interferometer Phys. Rev. B 91245419

[124] Cabart C, Roussel B, Fève G and Degiovanni P 2018 Taming electronic decoherence in one-dimensional chiral ballistic quantum conductors Phys. Rev. B 98155302

[125] Nagy I, Aldazabal I and Rubio A 2012 Exact time evolution of the pair distribution function for an entangled twoelectron initial state Phys. Rev. A 86022512

[126] Oriols X 2007 Quantum-trajectory approach to timedependent transport in mesoscopic systems with electronelectron interactions Phys. Rev. Lett. 98066803

[127] Buscemi F, Bordone P and Bertoni A 2011 On demand entanglement in double quantum dots via coherent carrier scattering New J. Phys. 13013023

[128] Buscemi F, Bordone P and Bertoni A 2008 Simulation of decoherence in 1d systems, a comparison between distinguishable- and indistinguishable-particle collisions Phys. Status Solidi c 5 139-42

[129] Buscemi F, Bordone P and Bertoni A 2007 Carrier-carrier entanglement and transport resonances in semiconductor quantum dots Phys. Rev. B 76195317

[130] Christov I P 2019 Time dependent spatial entanglement in atom-field interaction Phys. Scr. 94045401

[131] Hanbury Brown R and Twiss R Q 1956 Correlation between photons in two coherent beams of light Nature 177 27-9

[132] Kiesel H, Renz A and Hasselbach F 2002 Orbital entanglement and violation of Bell inequalities in mesoscopic conductors Nature $\mathbf{4 1 8} 392$

[133] Büttiker M 1990 Scattering theory of thermal and excess noise in open conductors Phys. Rev. Lett. 65 2901-4

[134] Büttiker M 1992 Flux-sensitive correlations of mutually incoherent quantum channels Phys. Rev. Lett. 68 843-6

[135] Büttiker M, Samuelsson P and Sukhorukov E V 2003 Entangled Hanbury Brown Twiss effects with edge states Proc. Int. Symp. Quantum Hall: Past, Present and Future; Physica E 20 33-42

[136] Bell J S 1964 On the Einstein Podolsky Rosen paradox Phys. Phys. Fiz. 1 195-200

[137] Samuelsson P, Sukhorukov E V and Büttiker M 2003 Orbital entanglement and violation of bell inequalities in mesoscopic conductors Phys. Rev. Lett. 91157002

[138] Chen Y-J and Xiao J-L 2016 Influences of the temperature on the parabolic quantum dot qubit in the magnetic field Low Temp. Phys. 186241

[139] Samuelsson P, Neder I and Büttiker M 2009 Entanglement at finite temperatures in the electronic two-particle interferometer Phys. Scr. T137 014023 
[140] Ingber L, Pappalepore M and Stesiak R R 2014

Electroencephalographic field influence on calcium momentum waves J. Theor. Biol. 343 138-53

[141] Bertoni A and Goldoni G 2007 Phase lapses in scattering through multielectron quantum dots: mean-field and fewparticle regimes Phys. Rev. B 75235318
[142] Pawłowski J, Górski M, Skowron G and Bednarek S 2017 Generation of Schrödinger cat type states in a planar semiconductor heterostructure Phys. Rev. B 96115308 